\title{
ENERGY EFFICIENT OPERATION OF A BASE TRANSCEIVER STATION USING MARKOV DECISION PROCESS
}

\author{
by
}

Udaya Raj Regmi

Bachelor of Science in Electronics and Communication Engineering American International University - Bangladesh (AIUB), 2002

\author{
A thesis \\ presented to Ryerson University \\ in partial fulfillment of the \\ requirements for the degree of \\ Master of Applied Science \\ in the Program of \\ Electrical and Computer Engineering
}

Toronto, Ontario, Canada, 2017

(C)Udaya Raj Regmi, 2017 
I hereby declare that I am the sole author of this thesis.

I authorize Ryerson University to lend this thesis to other institutions or individuals for the purpose of scholarly research.

I further authorize Ryerson University to reproduce this thesis by photocopying or by other means, in total or in part, at the request of other institutions or individuals for the purpose of scholarly research.

I understand that my thesis may be made electronically available to the public. 


\section{Abstract \\ Energy Efficient Operation of a Base Transceiver Station using Markov Decision Process \\ Udaya Raj Regmi, 2017 \\ Master of Applied Science \\ Electrical and Computer Engineering \\ Ryerson University}

Around $3.15 \%$ of the total world energy consumption accounts for Information and Communication Technology (ICT) sector. ICT's contribution to global greenhouse gas emission is expected to double in the coming five years.

Base transceiver station (BTS), an important but energy hungry component of access network in a cellular communication system, is usually resourced to serve busy hour traffic but remains under-utilized for most of the 24-hour period irrespective of the traffic load. Hence, self organizing networks (SON) that react to the variable traffic load are being studied to minimize energy consumption without compromising the QoS of the network.

Discrete time Markov decision process (DTMDP) as an optimization tool to manage the operation of BTS is investigated in this thesis. MDP finds an optimal policy that takes state specific optimal decisions, i.e. actions, and gets immediate rewards which maximizes the long term expected reward. The rewards obtained are the power savings when BTS operates as a SON by means of dynamic sectorization against the operation of BTS in uppermost mode irrespective of the traffic load. Further, transition cost to address mode switching cost and delay cost to address QoS are also discussed and elaborated through appropriate simulations to realize the actual energy savings. 


\section{Acknowledgments}

I wish to acknowledge those who I feel have greatly aided me in completing this thesis.

I would like to thank Professor Lian Zhao for providing an open study and discussion environment, having faith in me through out the project, constant encouragement and willingness to provide advice, and very clear guidance towards the success of this thesis project.

Special thanks to my colleagues in Ryerson Communication lab, specially Mushu Li, without whose help I would not have been able to get through this difficult and emotional time. Thanks to all friends who ever helped me over the past two years.

Special thanks go to my family especially my mom and dad, and my wife and daughter for their constant support. 


\section{Table of Contents}

$\begin{array}{ll}\text { Title Page } & \text { i }\end{array}$

Author's Declaration ii

$\begin{array}{lll}\text { Abstract } & \text { iii }\end{array}$

Acknowledgements $\quad$ iv

Table of Contents $\quad$ v

List of Tables $\quad$ viii

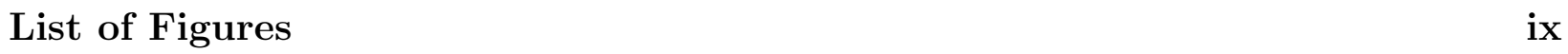

1 Introduction $\quad 1$

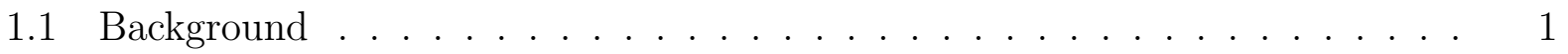

1.2 Literature Review . . . . . . . . . . . . . . . . . . . . . . . . 4

1.3 Problem Formulation . . . . . . . . . . . . . . . . . 7

1.4 Research Contribution . . . . . . . . . . . . . . . . . . . 7

1.5 Thesis Outline . . . . . . . . . . . . . . . . . . 9

2 Basic Theoretical and Conceptual Framework 10

2.1 Base Station and Cellular Architecture . . . . . . . . . . . . . . . 10

2.1.1 Omni Sector Configuration . . . . . . . . . . . . 11 
2.1 .2 Two Sector Configuration . . . . . . . . . . . . . . . . . . . 12

2.1 .3 Three Sector Configuration _. . . . . . . . . . . . . . 13

2.1.4 Six Sector Configuration . . . . . . . . . . . . . . . . . . 14

2.2 Operating Mode/Configuration of a BTS . . . . . . . . . . . . 14

2.3 Energy Consumption and Efficient Operation of a BTS . . . . . . . . 17

2.4 Markov Decision Process . . . . . . . . . . . . . . . . . . . . 18

$\begin{array}{llr}3 & \text { System Model } & 20\end{array}$

3.1 Network Model . . . . . . . . . . . . . . . . . . . . . . . . . 20 20

3.2 Traffic Model . . . . . . . . . . . . . . . . . . . . . . 22

3.3 Power Consumption Model . . . . . . . . . . . . . . . . . . . . . . . . 23

4 Proposed Markov Decision Process 26

4.1 Defining MDP States . . . . . . . . . . . . . . . . . . . . . 26

4.2 Possible MDP Actions . . . . . . . . . . . . . . . . . . . . . . . . 29

4.3 Analysis of MDP Decision Epoch . . . . . . . . . . . . . . . 30

4.4 Transition Probability Matrix Between States . . . . . . . . . . . 30

4.5 Reward Function Analysis . . . . . . . . . . . . . . . . . . . 34

4.6 MDP Optimization Problem Analysis . . . . . . . . . . . . . . . 36

5 Simulation/Numerical Results and Analysis $\quad 39$

5.1 Power Consumption and Saving Analysis . . . . . . . . . . . . . . . 41

5.1.1 Power Consumption and Saving Analysis Without Delay Cost and Transition Cost . . . . . . . . . . . . . . . 41

5.1.2 Power Consumption and Saving Analysis With Transition Cost . . . 43

5.1.3 Power Consumption and Saving Analysis With Delay Cost . . . . . 45

5.2 BTS Mode Distribution Analysis . . . . . . . . . . . . . . . . 46

5.2.1 Mode Distribution Analysis Without Delay and Transition Cost . . . 46

5.2.2 Mode Distribution Analysis With Transition Cost . . . . . . . . . 47

5.2 .3 Mode Distribution Analysis With Delay Cost . . . . . . . . . . 48 
5.3 Action Distribution Analysis . . . . . . . . . . . . . . . . . . 49

5.3.1 Action Distribution Analysis Without Delay and Transition Cost . . 49

5.3.2 Action Distribution Analysis With Transition Cost . . . . . . . . 50

5.3.3 Action Distribution Analysis With Delay Cost . . . . . . . . . . . 51

5.4 Integrated Analysis . . . . . . . . . . . . . . . . . . . . . 51

5.5 Analysis on Effect of Epoch Time . . . . . . . . . . . . . . . . . 53

6 Conclusions and Future Works $\quad 54$

6.1 Summary . . . . . . . . . . . . . . . . . . . . . 54

6.2 Main Contributions . . . . . . . . . . . . . . . . . 55

6.3 Future Works . . . . . . . . . . . . . . . . . . . 56

$\begin{array}{lr}\text { A Abbreviation List } & 58\end{array}$

$\begin{array}{lr}\text { Bibliography } & 59\end{array}$ 


\section{List of Tables}

5.1 Simulation Parameters . . . . . . . . . . . . . . . . . . . . . 40 


\section{List of Figures}

1.1 Power Consumption Breakdown in Cellular Network [6]. . . . . . . . . . . 3

2.1 Network Layout . . . . . . . . . . . . . . . . . . . . . 11

2.2 Operating in Omni Mode . . . . . . . . . . . . . . . . 12

2.3 Operating in Two Sector Mode . . . . . . . . . . . . . . 13

2.4 Operating in Three Sector Mode . . . . . . . . . . . . . 13

2.5 Operating in Six Sector Mode . . . . . . . . . . . . . . . 14

2.6 Reconfiguration of six sector mode to Omni, 2S, 3S and $6 \mathrm{~S}$ in (a), (b), (c) and (d) respectively . . . . . . . . . . . . . . . . . . . . . 16

2.7 Week Long Traffic Variation Including Holiday [33] . . . . . . . . . . . . . 17

3.1 Proposed Network Model with Variable-sector Base Station . . . . . . . . . . 21

3.2 Typical Cell Site Components of a BTS [34] . . . . . . . . . . . . . . . 23

4.1 Birth Death Process . . . . . . . . . . . . . . . . . . . . 27

4.2 Sampled Time Approximation to Markov Process . . . . . . . . . . . . . . 31

4.3 Grouped Transition Probability Matrix of a Markov Process . . . . . . . . . 33

5.1 Normalized Traffic Load of a Typical Weekday. [33] . . . . . . . . . . . . . . 41

5.2 Daily Power Consumption With and Without MDP. Max $\lambda=1000$, Transition Cost $=0$, Delay Cost $=0$, Epoch $=1$ s . . . . . . . . . . . . . 42

5.3 Daily Power Saving. Max $\lambda=1000$, Transition Cost $=0$, Delay Cost $=0$, Epoch $=1$ s . . . . . . . . . . . . . . . . . . . . 43 
5.4 Daily Power Consumption Analysis With and Without MDP. Max $\lambda=1000$, Transition Cost $=1000$, Delay Cost $=0$, Epoch $=1$ s $\ldots \ldots \ldots 44$

5.5 Daily Power Saving With and Without MDP. Max $\lambda=1000$, Transition Cost $=1000$, Delay Cost $=0$, Epoch $=1 \mathrm{~s} \ldots \ldots \ldots \ldots 44$

5.6 Daily Power Consumption Analysis With and Without MDP. Max $\lambda=1000$, Transition Cost $=0$, Delay Cost $=100$, Epoch $=1$ s $\ldots \ldots \ldots 45$

5.7 Daily Power Saving. Max $\lambda=1000$, Transition Cost $=0$, Delay Cost $=100$,

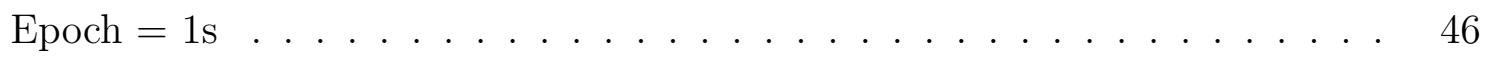

5.8 BTS Mode Distribution Analysis. Max $\lambda=1000$, Transition Cost $=0$, Delay Cost $=0$, Epoch $=1$ s $\ldots \ldots \ldots \ldots \ldots \ldots \ldots \ldots$

5.9 BTS Mode Distribution Analysis. Max $\lambda=1000$, Transition Cost $=1000$,

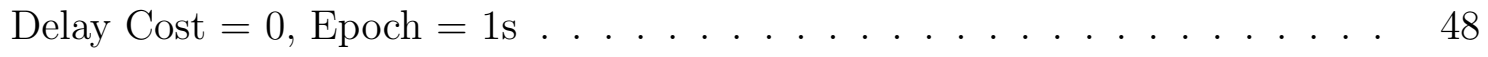

5.10 BTS Mode Distribution. Max $\lambda=1000$, Transition Cost $=0$, Delay Cost $=$ 100, Epoch $=1$. . . . . . . . . . . . . . . . . . 49

5.11 Action Distribution. $\operatorname{Max} \lambda=1000$, Transition Cost $=0$, Delay Cost $=0$,

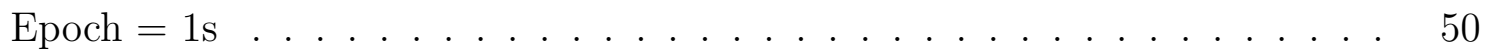

5.12 Action Distribution Analysis. Max $\lambda=1000$, Transition Cost $=1000$, Delay Cost $=0$, Epoch $=1 \mathrm{~s} \ldots \ldots \ldots \ldots \ldots \ldots$

5.13 Action Distribution. $\operatorname{Max} \lambda=1000$, Transition Cost $=0$, Delay Cost $=100$,

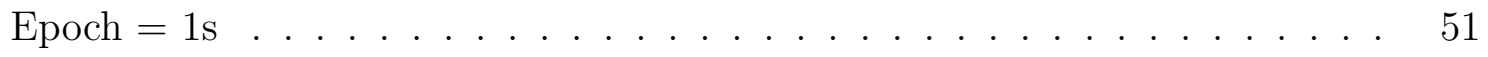

5.14 Total Power Saving Variation against Time Epochs . . . . . . . . . . . 53 


\section{Chapter 1}

\section{Introduction}

\subsection{Background}

With the advent of smart-phones and tablet devices, the demand for high speed communications has grown significantly in the last few years. Keeping that in mind, the research has also focussed on providing as much data rate as possible. Even though the network envisioned to address the demand of high speed communication serves its purpose during the day, it remains idle for most of the time during the 24 hour period, especially at night, i.e., the resource is under-utilized, and at the same time, energy is wasted to keep the network fully ON just to provide coverage. The impact has two-folds, economic burden as well as the environmental issue. On the economic aspect, the under-utilized resource is one of the most important cost component for the operators, i.e., operating expenditure (OpEx), which will be eventually passed on to the consumers. Furthermore, with respect to the environmental concern, it is also a major contributor of the greenhouse gas emission which is expected to increase continuously in the coming years, as more networks will be rolled out. ICT is being used as a tool to reduce the carbon footprint from different forms of industry like farming, aviation, production, transportation, etc. However, ICT itself has been stated as the significant contributor to greenhouse gas emission and its contribution is quite noticeable at

around 2 percent at present [1]. This percentage is expected to increase in future with the 
evolution of the concept that everything being connected.

However, the issue of energy saving is steadily gaining pace in the last few years, thereby reducing the impact on greenhouse gas emissions. The reduction in energy usage also brings a great relief to the operators in terms of OpEx reduction. The study has shown that OpEx of the operators is three times the capital expenditure (CapEx). The component of energy expenditure in that OpEx is at least 32\% in the emerging market like India and around 18\% in the mature European market [2].

The earliest study regarding energy issues in the fixed network can be attributed to [3] where the authors highlighted the importance of the sleep modes on network protocols by investigating the energy consumption in Internet devices. Several studies have been carried out focussing on energy efficiency (EE) aspects of wireless communications, not only through individual collaboration between the researchers, but also through research projects such as WINNER [4], EARTH [5], Green Radio [6], OPERANet [7], eWIN [8], 3GPP [9], and TREND [10]. Against the existing trend of EE study on improving the efficiency of hardware components and operating the network against the variable load, researchers are even exploiting the previously unexplored areas like increasing EE through caching of data at base stations [11]. As future networks are expected to be data hungry, the study highlights that caching at BTS with the use of power efficient hardware increases EE, specifically when interference level is low, the back-haul capacity is stringent, and the content popularity distribution is skewed. These highlight the importance being given to the energy efficient operation of wireless communication systems. 


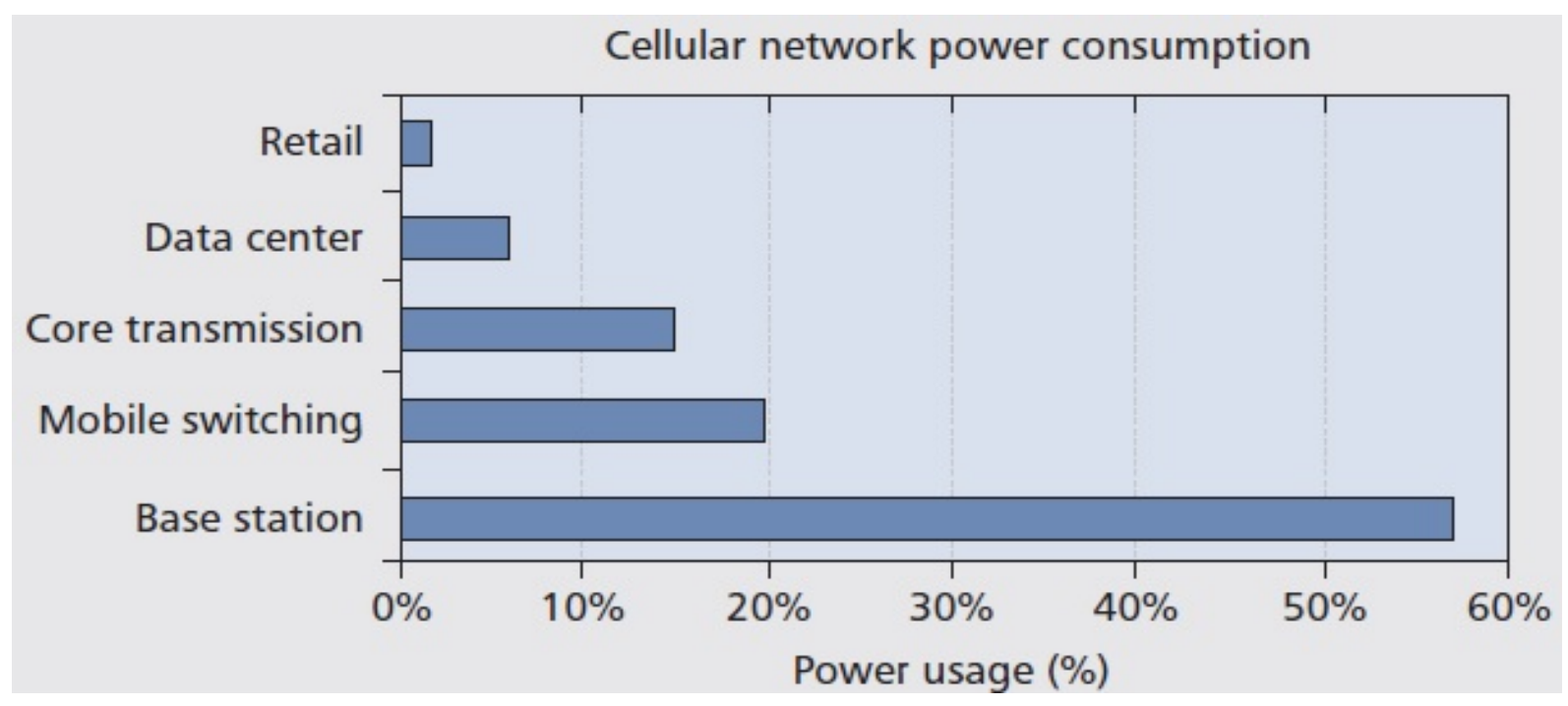

Figure 1.1: Power Consumption Breakdown in Cellular Network [6].

Energy is consumed by different network components of a cellular network which is broadly classified as core network and access network. Through examination, it has been shown that bulk of the energy consumption, i.e., almost $80 \%$ [6], is in the radio access network which consists of base station controller (BSC) and BTS as shown in Fig. 1.1. Out of these two, BTS consumes more energy as it comprises the most energy hungry components of the whole network like radio transceivers, transmit antennas, cooling equipment, baseband processors, power amplifiers, and so on. Likewise, the energy consumption of the network and switching subsystem (NSS) is comparatively less in comparison to radio subsystem (RSS), of which BTS is a part, as BTS installation has outnumbered switching system these days. It has hence attracted attention of researchers who have identified significant potential for energy conservation in this area [12]. Further, energy consumption also depends on the type of BTS being deployed, i.e., Macro, Micro, Pico, or Femto cells, and the location being deployed, such as urban, sub-urban or rural. Hence, the energy saving techniques being considered are not supposed to give similar energy saving returns across all types and terrains of BTS deployment.

The telecom network is dimensioned taking into consideration the peak time or busy hour traffic. However, such busy hours occur only for a few hours throughout the day which 
might vary depending on time and area of deployment. This means, most of the time, the resource that is provisioned to run a network remains idle, most prominently during night when traffic is very low. So, it provides the opportunity of energy savings when the network is configured to changing traffic demand. This not only allows to grasp the energy saving opportunity provided by the typical traffic pattern, but also addresses the change in traffic arrival pattern brought by the unseen circumstances such as natural calamities, social gatherings, sporting activities, etc. However, the techniques proposed in several studies also have shortcomings such as coverage holes, higher outage probability, degradation of quality of service (QoS), network disruption, added interference, to name a few that has been neglected or not properly addressed in the studies as highlighted by the survey on sleep-mode techniques in [12]. In addition to that, some proposed techniques increase EE of the network at the cost of the rapid decrease in the battery level of a mobile station (MS) when the distance between the MS and the transceiver is increased or by the creation of coverage holes. This is because the MS keeps on searching for the available network when it falls in coverage hole or increases its transmission power to maintain required signal to interference plus noise ratio (SINR) level when BTS transceiver is far away, thereby draining the battery power really fast. Therefore, there is a trade-off between EE and other QoS parameters, which should be carefully addressed to reap the net benefit. Power savings can be measured through different parameters such as energy consumption ratio (ECR), throughput power consumption (TPC), and area power consumption (APC).

\subsection{Literature Review}

Approaches to realize EE in cellular wireless networks can be broadly classified into the improvement on hardware components, sleep mode techniques, optimization in radio transmission process, network planning and deployment, and adoption of renewable energy resources. Each of them has its own share of advantages and limitations [12]. In this study, we first review those literatures whose focus is on the efficient management of the currently deployed 
networks to increase their EE.

Studies on EE of wireless communication have proposed several mechanisms such as cell size adjustment [13] [14], dynamic base station switching [15] [16], dynamic reconfiguration of BTSs [17], base station sleeping [1] [18] - [20] etc. Authors in [13] show that it is possible to adaptively adjust the cell size according to fluctuating traffic load, and large amount of energy can be saved when traffic load is low. Authors in [14] proposed three novel cell zooming techniques that dynamically adjust the transmission power, hence the coverage area, depending on the location of the farthest user. In [15], authors proposed an on-line distributed controller that takes into consideration the effect to neighbouring base stations when a base station is switched OFF and gradually switch OFF base stations to minimize its effect on the whole network. Minimum energy cost problem (MECP) has been formulated in [16] by considering the switching cost of dynamically switching ON/OFF base stations in cellular networks, therby minimizing the total energy cost of BTS. Dynamic cell reconfiguration framework has been proposed in [17] that jointly tackles the problem of base station selection, transmit power budget adaptation, and user association in cellular network which achieve significant power savings at low load and considerable saving at high load.

Base station sleeping strategies along with small cell deployment techniques have been studied in [1] by formulating the power consumption minimization and EE maximization problem which confirmed the effectiveness of the sleeping strategy, but efficiency of which was dependent on the type of sleeping strategy being used. As the efficiency of the small cells in meeting the increasing demand for high-date-rate wireless applications being shown viable, authors in [18] have studied small cell random and strategic sleeping policies under constraints on coverage probability and wake-up times. The findings showed $30 \%$ improvement in EE for random sleep strategy and further $15 \%$ for strategic sleeping policy. Optimization of base station density configuration is studied in [19] in improving EE of the cellular network with traffic aware sleep strategies. The study also claims that sleeping strategy not only saves energy but also improves the quality of radio links. Authors in [20] proposed a scheme that jointly decides the sleep decision of a femto BTS and the user association 
between active BTSs at times of low traffic to reduce the total energy consumption of the open-access femto cell network.

Studies [23] - [25] show that dynamic reconfiguration of BTS sectors in tandem with the variation in traffic load gives higher energy savings. In line with the proposed energy-saving techniques in EARTH project to minimize the power consumption of mobile networks by $50 \%$ [25], authors in [23] demonstrated that underutilized 3-sector BTS can be reconfigured to omni-cell BTS without significantly degrading the service in a network thereby saving the power of two out of three radio units on a typical 3-sector BTS. In [24] it was shown that the traffic-sensitive dynamic sectorization of BTS in OFDMA-based cellular access networks has the potential to reduce energy consumption. Likewise, in [26] authors proposed a novel energy-aware dynamic sectorization strategy where some sectors of the base stations are switched OFF, and the beam-width of the remaining sectors are reconfigured to cover the area vacated by the switched OFF sector in adaptation to the temporal traffic variations.

Markov Decision Process (MDP), which is a discrete time stochastic control process, initially conceptualised by [31] has been used in variety of fields to solve optimisation problems [32]. In line with that, some researchers have proposed different schemes for managing wireless networks using MDP as an optimization tool [27] [28] [29]. In [27] authors derived dynamic decision rule using MDP to switch off relay nodes at times of low utilization in relay-aided cellular networks and proved the superiority of the proposed scheme by comparing against the always ON policy and some threshold based policy. Authors in [28] proposed a MDP based policy to minimize the long term network power consumption by shutting down a BTS at times of low traffic and serving the area covered by that cell through the increased transmission from the adjacent cells. Study in [29] proposed an MDP based BTS management scheme that manages the group of base stations against the time varying traffic in self-organizing networks, which captures the dynamics of the handover traffic as well. While developing the transfer actor-critic algorithm, the learning framework for BTS energy saving, the authors in [21] formulated the BTS switching operation under varying traffic load as MDP. 


\subsection{Problem Formulation}

This study involves the EE analysis of macro BTS operation in line with the variation in traffic demand. Rather than allowing the whole BTS site to go into sleep mode, the sectored configuration of the BTS site is altered in accordance with the traffic variation in the coverage area. This addresses the issue of coverage holes and larger distance between transceiver and MS in the coverage area. Consequently, it also helps to maintain the transmission guidelines stipulated by the regulatory authorities with regard to set maximum transmit power of a BTS transceiver. Cell site might go into sleep mode only when there is no traffic in the coverage area and is allowed by the policy in effect. Starting with the operation of the cell in omni mode, it might move to 2-sector mode or 3-sector mode or finally to 6-sector mode in the increasing order of traffic intensity under its coverage area or vice-versa in the decreasing order of traffic intensity from 6-sector mode to switch OFF mode, i.e., sleep mode. The cell modes can be switched to any of the adjacent modes depending on the traffic fluctuation and MDP policy.

Hence, in order to avoid the frequent switching of the cell between states and to reduce power consumption, a policy to optimize the long-term power consumption needs to be developed. The main goal of this study is therefore to develop a mechanism for minimizing the long-term expected power consumption of the BTS network. For this purpose, a Finite Markov Decision Process (FMDP) is developed as a tool to operate at a BTS site in an energy efficient manner, and the efficacy of such optimization policy is demonstrated through simulation results and numerical analysis.

\subsection{Research Contribution}

The main contribution of this research is to use a Markov Decision Process (MDP) as an optimization tool to improve the efficiency of BTS operation in an energy efficient manner. In line with that, in this study, MDP has been used to devise a policy that efficiently operates a base station which gives maximum long term average reward in terms of energy consumption, 
i.e., long term minimum average energy consumption.

Likewise, the effect of transition cost in BTS management has been studied as well. Transition of modes, i.e., switching the sectors $\mathrm{ON}$ or OFF, comes at some cost due to user handover, change of antenna configuration, processing time, etc. The effect of such cost while managing BTS is also analysed such that the efficiency achieved through the proposed scheme matches with the practical deployment outcomes.

Furthermore, the effect of delay cost is also taken into account. This is the cost that the system will incur when it has to make the call request wait before it is served. Incorporation of such cost in taking appropriate decision helps improve the quality of the service provided by the network, albeit at the cost of high energy consumption.

Finally, effect of decision epoch time in the realization of energy savings has been considered. Epoch time is the time interval that MDP process observes the system state and takes appropriate decision, i.e., takes action. Duration of this time epoch plays an important role in the efficiency of the network to react to time varying traffic, which will have significant impact on the actual realization of energy savings.

Although the model looks simple, it alleviates few concerns regarding QoS and transition cost which are ignored together or individually in literature. The most prominent feature is that it is scalable, i.e., integrated consideration of epoch time along with transition cost and delay cost allows the network controller to choose to operate the site with trade-off between processing overheads, computational complexity, frequent switching of modes and EE. Further, coverage area of BTS site is not altered, which addresses the issue of coverage holes and unnecessary draining of battery power of user terminals. The inherent property of MDP avoids frequent switching of BTS site which has been ignored in some studies with threshold based energy optimization techniques. 


\subsection{Thesis Outline}

The remainder of this thesis is organized as follows. Chapter 2 deals with the basic theoretical and conceptual background on different modes of communication in cellular communication system. Furthermore, this section also deals with the process on how energy consumption minimization can be achieved and the opportunities where the energy saving can be exploited. Theoretical idea behind Markov Decision Process is also discussed in this chapter.

Chapter 3 deals with the system model being evaluated in this study. The underlying models such as network model, traffic model, and power consumption model are presented in detail.

Chapter 4 deals with MDP in detail relevant to this study by introducing basic tuples of MDP such as states, actions, rewards, transition probability, discount factor and MDP decision epoch time, all of which are important constituents of the proposed approach. This chapter elaborates the proposed concepts in detail.

Chapter 5 deals with the numerical results and analysis of the study.

Chapter 6 presents the summary of this study. This last chapter also contains the analysis of the shortcomings of the study and sheds some light on future studies as well as main contributions. 


\section{Chapter 2}

\section{Basic Theoretical and Conceptual Framework}

\subsection{Base Station and Cellular Architecture}

Base Transceiver Stations (BTSs) is an integral part of a radio access network (RAN) that facilitates radio communication between a mobile user equipment and core network in a cellular communication system. The area around the BTS is often modelled as a hexagonal structure known as cell. Depending on the underlying mobile communication technology, coverage area, traffic load, subscriber behaviour and other radio transmission parameters, the cell may vary in size. BTS communicates with other stand-alone network such as fixed line network, via base station controller (BSC) and mobile switching center (MSC). Rather than going into details of other aspects of cellular communication networks, we will deal with the BTS configuration in terms of sectorization in this section. BTS sectorization is an important constituent of this research work as we propose to alter the configuration of the BTS in order to save energy. 


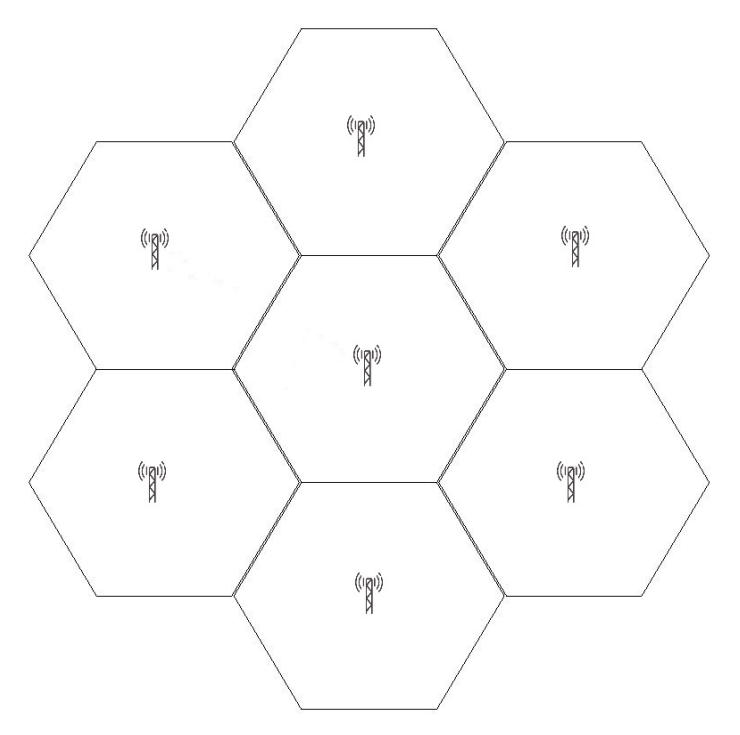

Figure 2.1: Network Layout

The cells are arranged in honeycomb structure as shown in Fig. 2.1. Hexagonal cells are preferred over other cell configuration primarily due to their large coverage area, hence less number of cells and most importantly they neither overlap nor leave any gaps between them and closely cover the circular area around the cell in ideal case. In terms of deployment, cells are either omni-directional or sectoral, depending on the radiation pattern of the transmitting antenna. Hence, a single site may consist of more than one cell/sector per site with each cell having their own physical cell id. However, in this study, BTS operation is controlled individually by a distributed controller that resides in each BTS site without coordination among BTSs, thus making the operation simpler without having the requirement of overheads to exchange network information between them. Details on the configuration/modes of BTS are discussed in the following sections.

\subsubsection{Omni Sector Configuration}

A BTS will be operating in an omni sector configuration when a single BTS antenna situated at the center of the cell receives and radiates signal uniformly in all the directions of the antenna in a two dimensional horizontal plane, i.e., $360^{\circ}$ around the antenna which is also the beam-width of the antenna as shown in Fig. 2.2. The doughnut shaped radiation pattern 
is the hallmark of this type of cell configuration. Also known as non-directional antenna because of its uniform transmission in all directions, such antenna configuration is used when it is required to serve the area when there is low subscriber activity. Although it is possible to use single omni antenna to cover the $360^{\circ}$ area around the cell, operators even use sectored antenna for omni coverage because of the superior performance of the sectored antenna over omni antenna.

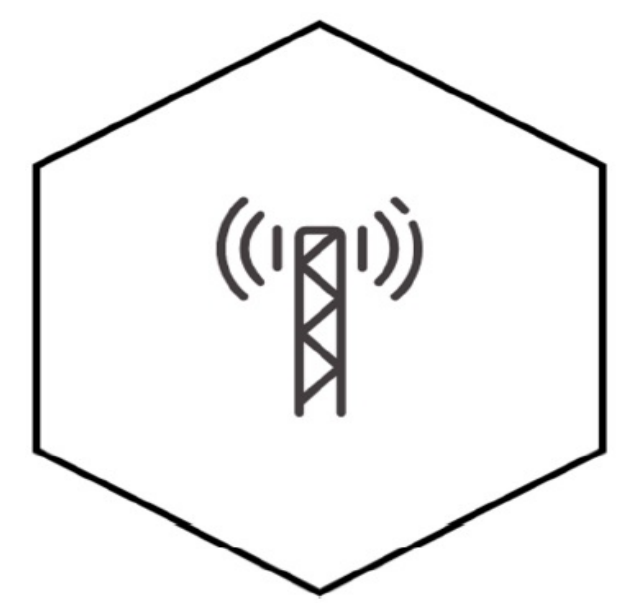

Figure 2.2: Operating in Omni Mode

\subsubsection{Two Sector Configuration}

In the two sector configuration, the coverage area of a BTS site is divided into two halves as shown in Fig. 2.3, hence the beam-width of this configuration is 180 degrees. This type of configuration is more popular in maintaining coverage in highways with boundaries perpendicular to the highway. However, such configuration is also one of the different configurations that has been proposed to be used in this study in order to minimise the energy consumption when site configuration is dynamically varied in line with traffic variations and MDP actions. 


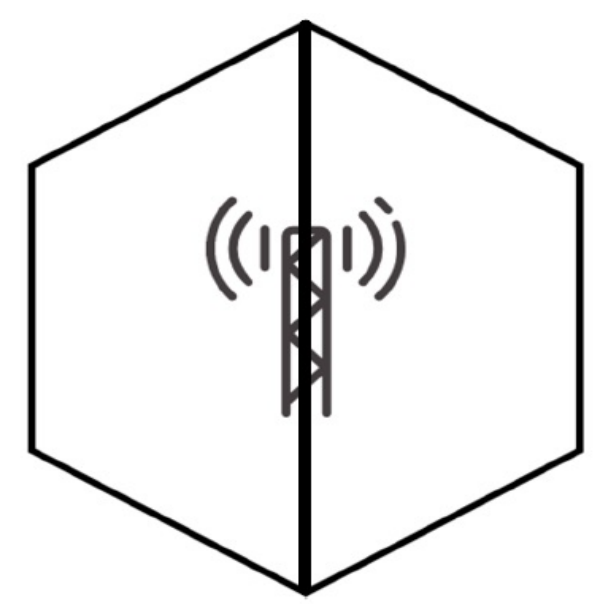

Figure 2.3: Operating in Two Sector Mode

\subsubsection{Three Sector Configuration}

This three sector configuration is the most popular form of sectorised implementation of a BTS site as shown in Fig. 2.4. The beam-width of the sectors in this configuration is 120 degrees. This mode is used to serve high traffic specially in $2 \mathrm{G}$ networks and $3 \mathrm{G}$ networks because of the high SINR between BTS and MS, thus facilitating high data rate to the users.

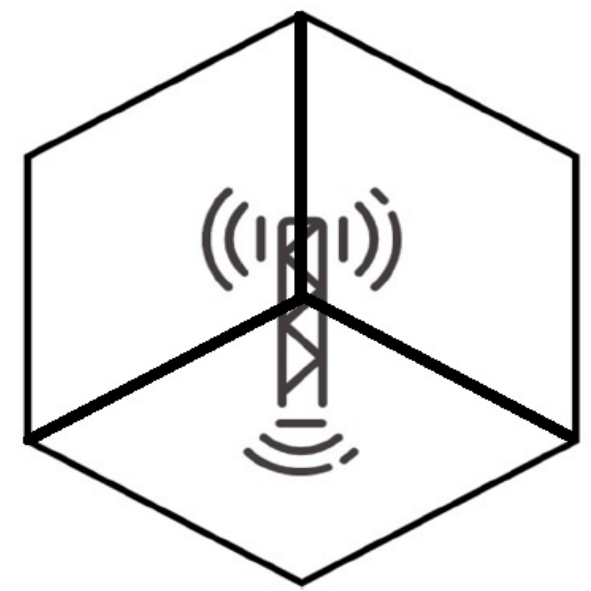

Figure 2.4: Operating in Three Sector Mode 


\subsubsection{Six Sector Configuration}

Six sector configuration is the requirement of today's network where it is required to serve data hungry high traffic volume, hence more popular in $3 \mathrm{G}$ networks and beyond. The beamwidth of the sectors in this configuration is generally 60 degrees, however it can be varied depending on the type of antenna being deployed. This type of higher order sectorization not only increases the overall data rate but also improves the network coverage as there is significant antenna gain from three sector deployment to six sector deployment [22]. The configuration of this network is shown in Fig. 2.5.

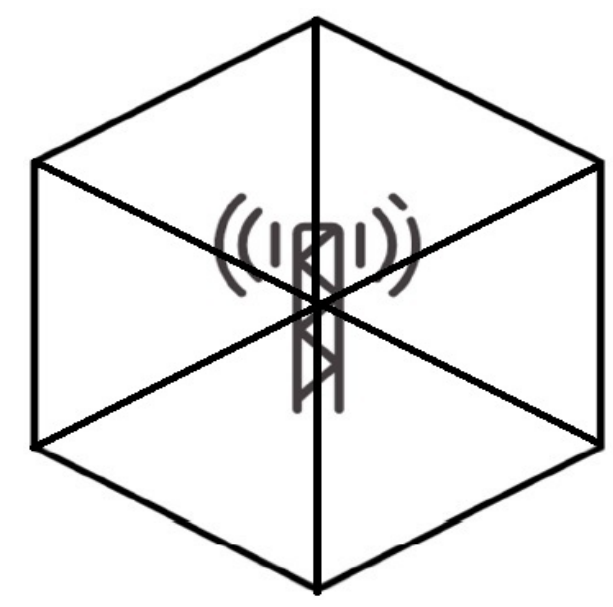

Figure 2.5: Operating in Six Sector Mode

\subsection{Operating Mode/Configuration of a BTS}

Dictated by the action chosen by the optimal MDP policy, BTS is made to operate in appropriate mode/configuration. The proposed strategy can be termed as adaptive sectorization where the BTS site is reconfigured to save energy. The mode/configuration chosen in this study are popular configurations presently in use in cellular communication networks, which are stated as below.

- Mode 1: When all sectors are turned OFF, BTS is said to be operating in 'sleep mode'. 
- Mode 2: When only one sector is turned ON serving all the area around the BTS site, it is said to be operating in 'Omni Mode'.

- Mode 3: When two sectors are turned ON, each sector serving each half $\left(180^{\circ}\right)$ area around the BTS site, it is said to be operating in 'Two sector (2S) mode'.

- Mode 4: When three sectors are turned ON, each sector serving the $120^{\circ}$ area around the BTS site, it is said to be operating in 'Three sector (3S) mode'.

- Mode 5: When all the six sectors are turned ON, each sector serving the $60^{\circ}$ area around the BTS site, it is said to be operating in 'Six sector $(6 \mathrm{~S})$ mode'

When the specific sector reconfiguration is required, the sectors are turned ON or OFF as per the requirement. In this case, the active sectors have to adjust their transmission beam width either by expanding or shrinking so that coverage is provided throughout the coverage area of a site. Several techniques exist to vary the beam width of the sector antenna three of which are enumerated as below:

- Finite beam switching using linear antenna arrays which can alter the beam-width to the required sector size or using a combination of a radiating dipole and a folded reflector to provide the required beam reconfigurability [30].

- Equipping BTS site with multiple sets of antennas i.e., set of 1,2, 3 or 6 antennas for omni, 2S, 3S, and $6 \mathrm{~S}$ mode respectively. These sets can then be activated or deactivated as per the requirement. In this case, set of three antennas can be used to serve in $3 \mathrm{~S}$ mode. This scheme eradicates the need to reconfigure the beamwidth of the antennas, specially when deploying $2 \mathrm{~S}$ mode [24].

- Deploying the BTS site as a physical six sector site. Then as per the requirement, the selected sectors can be activated or deactivated depending on the mode being chosen. As shown in Fig. 2.6 (a), sector antennas are configured for omni mode. In (b), sector A, B and C and D, E, and F combine to form $2 \mathrm{~S}$ mode. Likewise, in (c), A and B, C 
and $\mathrm{D}$, and $\mathrm{E}$ and $\mathrm{F}$ combine to form $3 \mathrm{~S}$ mode. In (d), six sectors serve their respective areas individually to form $6 \mathrm{~S}$ mode.

Other techniques, such as adaptive smart antenna employing linear antenna array and reconfigurable beam antennas for BSs with fanning capability, have also been discussed in the study [24] claiming that the proposed schemes can be practically implemented.

When two or more sectors, i.e., A to $\mathrm{F}$ as in Fig. 2.6 are combined to form other sector configuration, such combined sector antenna is fed through a single power amplifier (PA) or radio unit $(\mathrm{RU})$. Depending on the receive signal strength, the user handover has to be performed between sectors before each transition, i.e., mode change.

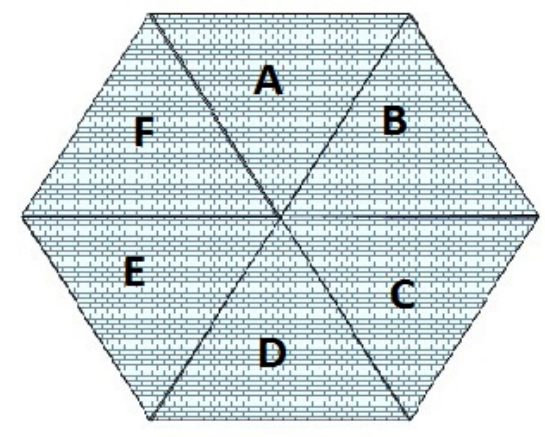

(a)

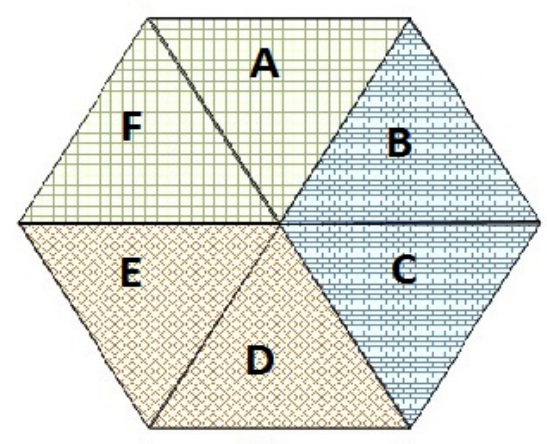

(c)

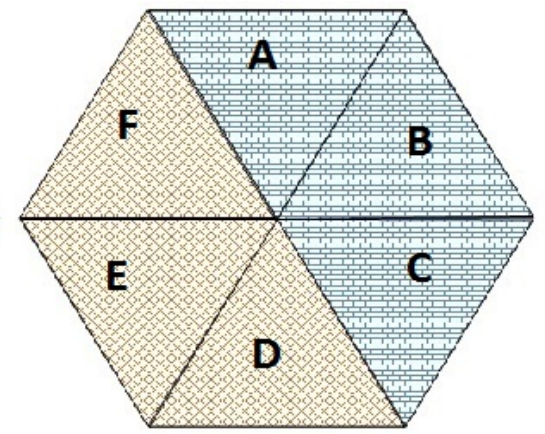

(b)

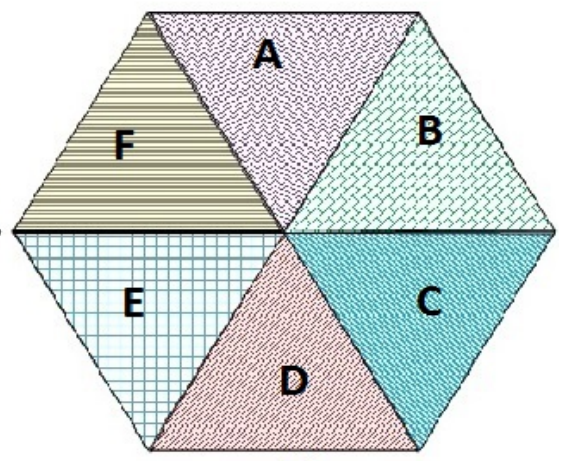

(d)

Figure 2.6: Reconfiguration of six sector mode to Omni, 2S, 3S and 6S in (a), (b), (c) and (d) respectively 


\subsection{Energy Consumption and Efficient Operation of a BTS}

As stated earlier, traffic is not time-homogeneous throughout the day. As such, resources are provisioned for the peak time traffic. This time-inhomogeneous nature of the telecommunication traffic along with idle resources present us with an opportunity to apply techniques to operate the BTS in an energy efficient manner. As shown in Fig. 2.7, the normalized traffic variation is clearly seen in all the days of the week. The traffic demand is maximum on the weekdays and significantly low during the weekend and in holidays. However, to maintain quality of service and availability of service, the network has to be provisioned that can handle peak time traffic. This means, the provisioned resource remains idle most of the time and unnecessary power consumption will continue if the proper mechanisms are not in place to address it.

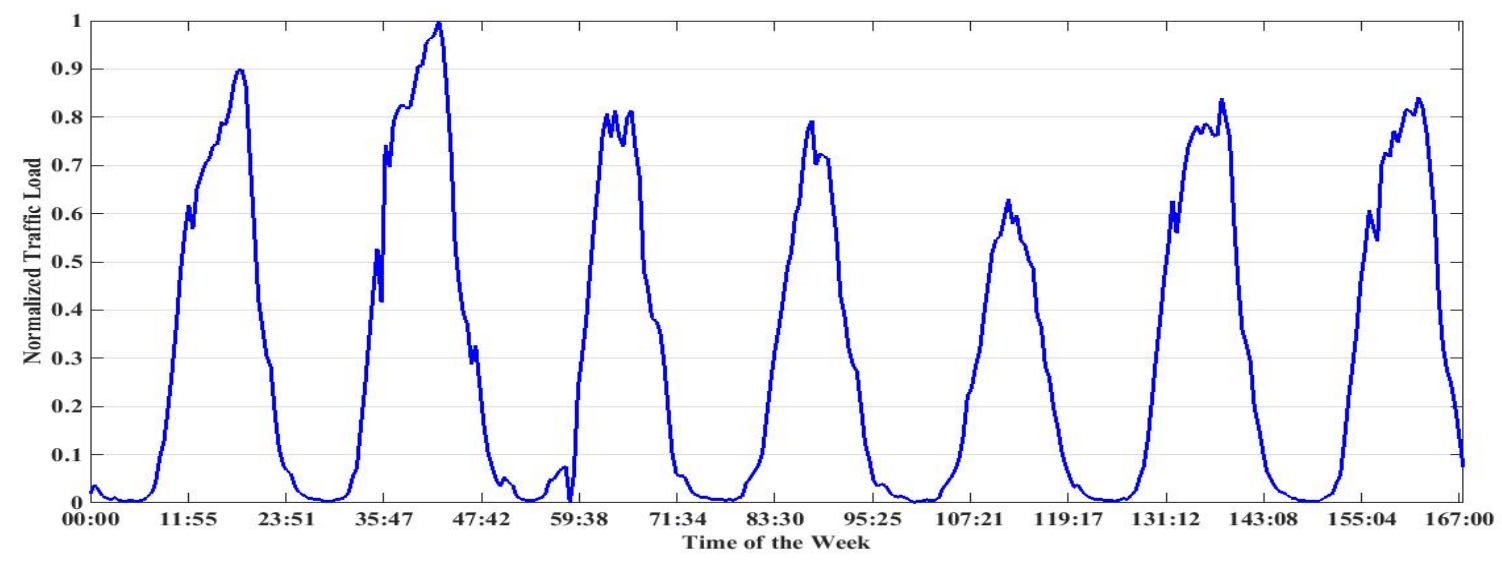

Figure 2.7: Week Long Traffic Variation Including Holiday [33]

Hence, it is the research focus of recent times to make network react to the changing traffic demand as shown in Fig. 2.7. Furthermore, efficient techniques in place also helps the network in responding to high traffic demand during festivals, events and other social gatherings. In this study, the proposed solution is to turn OFF sectors depending on the 
traffic variation guided by optimal MDP policy.

\subsection{Markov Decision Process}

MDP is a mathematical framework for modelling a decision making process where the outcomes are partly random and partly under the control of a decision maker. In other words, MDP is an optimization tool for decision making under uncertainty as stated in [31] and [32]. Most of the ideas expressed in this study in relation to MDP are mostly referenced from the above mentioned two literatures. A discrete time MDP (DTMDP) is a stochastic process, where at each time step, the process is in some state $s$, and the decision maker chooses some action $a$ that is available in that particular state. The process then responds at the next time step by randomly moving into a new state $s$ ' thereby giving the decision maker the corresponding reward $R_{\mathrm{a}}\left(s, s^{\prime}\right)$. The probability of moving to next state $s$ from $s$ depends on the transition probability stated as $P_{a}\left(s, s^{\prime}\right)$. State transition in MDP is expected to satisfy the Markovian property, i.e., given $s$ and $a$, the process should be conditionally independent of past states and actions.

The dynamics of the communication system in the cellular network, i.e., call arrival and departure, are modelled using a Markov chain, also known as birth-death process. Through this chain, real-time information on the channel occupancy of the system can be extracted, which is used as an input to the MDP model to identify the state of the system with call arrival and departure. The state of the process within the system keeps on changing, and appropriate actions are then taken to alter the dynamics of the system, i.e., changing of mode in this particular study. MDP is then employed here to take appropriate action for each state by employing MDP policy, which will give the highest long term average reward rather than immediate rewards. In this study, such rewards are related to the power savings when certain actions are taken.

Reverting to the basics, MDP is defined by a tuple $(\mathcal{S}, \mathcal{A}, \mathcal{P}, \mathcal{R}, \gamma, \mathcal{T})$, where, 
- $\mathcal{S}:=\left\{s \in S_{t} \mid t=1,2, \ldots, N\right\}$ is a finite set of possible states, where $S_{t}$ means the state of the system at time $t$.

- $\mathcal{A}$ is a finite set of possible actions, where $a_{\mathrm{s}, \mathrm{t}}$ means action $a$ taken by observing state $s$ at time $t$.

- $\mathcal{P}$ is a transition probability matrix $(\mathrm{TPM})$ between states $s\left(\mathrm{~S}_{\mathrm{t}}\right)$ to $s^{\prime}\left(\mathrm{S}_{\mathrm{t}+1}\right)$ denoted by $P\left(s^{\prime} \mid s, a\right)$.

- $\mathcal{R}$ is the reward obtained after taking action $a$, i.e., it is the immediate result of taking action $a$ at state $s$.

- $\gamma \in[0,1]$ is a discount factor. This value is used to represent the present value of future rewards.

- $\mathcal{T}:=\{1,2, \ldots, N\}$ where $N<\infty$, is the set of decision epoch time when an agent/controller looks into the system and takes appropriate decision.

Solving an MDP problem is finding an optimal policy, $\pi$, that maps the states to the actions, i.e., $\pi: S \rightarrow A$. In other words, given a state, the policy is to determine the corresponding action. MDP policy thus defines the behaviour of the agent that depends only on the current state and are stationary, i.e., when specific states are encountered by the controller, same action stated by the policy will be taken irrespective of time. A policy that gives the highest sum of the discounted future rewards of all the available policies is the optimum policy, $\pi^{*}$. Every MDP problem has an optimal policy, and such policy converts the MDP problem into a Markov chain with associated rewards [32].

In this study, optimum policy has been found through policy iteration algorithm. Starting with the first policy, $\pi_{0}$, up to the last available policy, $\pi_{\mathrm{n}}$, the algorithm exhaustively looks for the policy that gives maximum expected rewards, i.e., it converges to the optimum policy. This optimum policy, which is stationary and deterministic, is then expected to return the maximum reward that can be obtained from the system. 


\section{Chapter 3}

\section{System Model}

In this chapter, the descriptive overview of the network model, traffic model, and power consumption model being considered in this study are introduced along with the related assumptions.

\subsection{Network Model}

A typical deployment of macro base transceiver station (BTSs, eNB in LTE advanced network) as shown in Fig. 3.1 is considered. However, the internal layout of the BTS site is made to vary as shown in Fig. 3.1 in line with the change in the state of the system. The BTS site is configurable to one, two, three and six sectors/cells viz. omni, 2-sector, 3-sector or 6-sector mode. In the proposed scheme, the configuration of the BTS will be changed depending on the optimal policy developed by the MDP in order to save energy in the long term. The configuration of the BTS is changed accordingly to address the demand of the coverage optimized or capacity optimized mode. The BTS is said to be in sleep mode when no sector is turned ON. The BTS can only turn ON in omni-mode, i.e., single omni antenna

will serve all the users in the cell or remain in the same mode. When the channel occupancy ratio changes, the decision, i.e., action, is taken by the controller to switch the mode of the BTS to 2-sector mode, i.e., two sector antennas will now serve the users with corresponding 
changes to antenna configuration or switch back to omni mode or remain in the same mode.
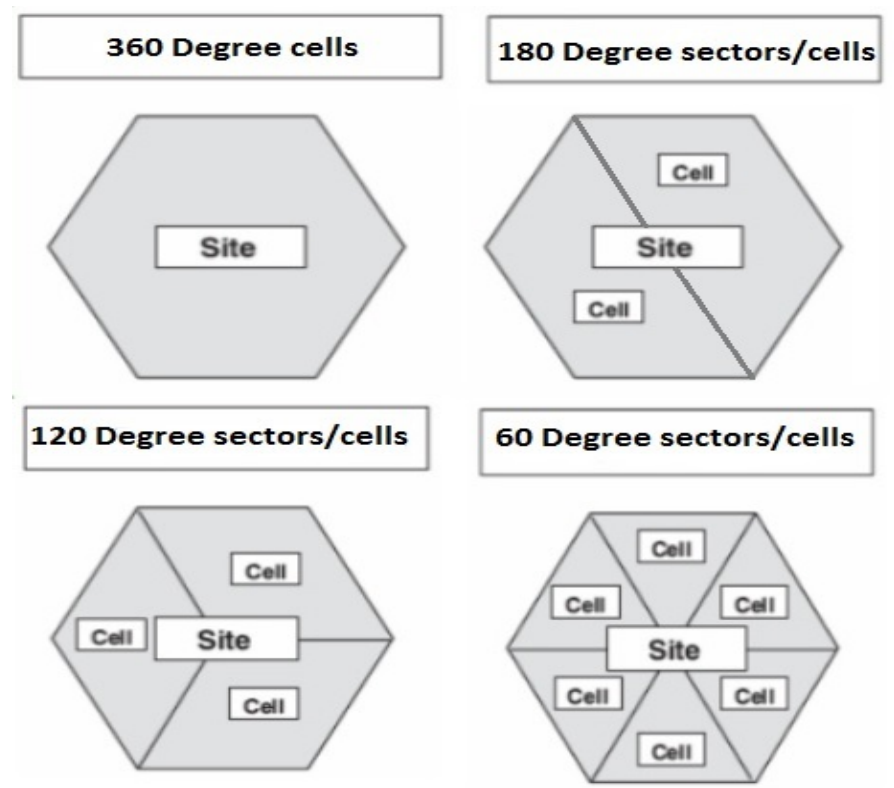

Figure 3.1: Proposed Network Model with Variable-sector Base Station

Likewise, similar approach is taken in 2-sector mode and 3-sector mode, i.e., either to switch to upper mode or lower mode or continue to remain in the current mode of BTS site configuration of sectors. However, in 6-sector mode, the controller can only choose to remain in the same mode or switch to lower 3 -sector mode. The lower mode are suitable for serving low traffic load and as the system moves to upper mode, higher traffic load can be served, i.e., 6-sector mode serving the highest traffic load. However, the controller that is supposed to take action does not only look to switch to upper mode to serve higher traffic load or switch to lower mode to serve lower traffic load. Instead, it looks if the long term expected reward is increased by taking such action. That means, the action being taken is dictated by MDP policy that looks to maximize the reward, i.e., higher energy saving or lower energy consumption. The proposed dynamic BTS management technique is distributed in nature, i.e., it is not centrally managed by the BSC. The distributed controller resides at the BTS site itself, and its performance is independent of the traffic variation or operating mode in the adjacent BTSs. This reduces the complexity of the BTS management as well as avoids 
the use of overheads to be exchanged between appropriate nodes of the communication system, either directly or through BSC. This simplifies the operation of the system under consideration and can be implemented into the system without much complexity.

\subsection{Traffic Model}

The coverage area around the BTS site is assumed to have homogeneous traffic in all the six sectors. The call arrival is modelled by Poisson distribution with mean rate $\lambda$, and the departure time is modelled by exponential distribution with mean time $1 / \mu$. The assumption in the scheme is that one call will be allocated to one channel. The probability that $x$ number of on-going calls in the system with the maximum number of channel being $N$ is given by Erlang's first formula or first Erlang distribution as,

$$
\operatorname{Pr}(X=x)=\frac{\frac{A^{x}}{x !}}{\sum_{k=0}^{N} \frac{A^{k}}{k !}},
$$

where,

$x$ is the number of occupied channels,

$A$ is the traffic intensity of the system given by the ratio $\lambda / \mu$.

In this study, the maximum number of occupied channels per sector/cell is $c$, hence there are total of $6 c$ channels available in a site when six sectors are turned ON. The corresponding steady state probabilities of channel occupancy can be obtained using (3.1) where $x$ varies from 0 to $6 c$.

The term, $A$, can also be taken as the average total offered traffic, which is the sum of the traffic injected by each user in the coverage region. The mode switching decisions are taken as per the optimal policy guided by the traffic condition in the coverage area. $P(6 c)$ is the probability that all the available channels are occupied. It is the grade of service (GoS) measure of the system, which gives the probability of all the channels being occupied. Hence,

for a particular required GoS, the traffic intensity offered by each user and the number of channels in use, total offered traffic intensity $A$ can be found through Erlang table, thereby 
finding the number of users that can be supported in a coverage area.

\subsection{Power Consumption Model}

A typical cellular communication system can be broadly classified into two components viz. core and access. The focus of this study is on the energy consumption of the BTS which is one of the major components of the access network along with the BSC. BTS, depending on whether it is macro, micro, pico or femto, consists of a number of Transceivers (TRXs). Typical components of a BTS TRX comprise a number of components such as AC-DC converter, i.e., main supply, active cooling equipment, DC-DC converter, baseband (BB) interface, RF transceiver, power amplifier (PA), and antenna interface as shown in Fig. 3.2

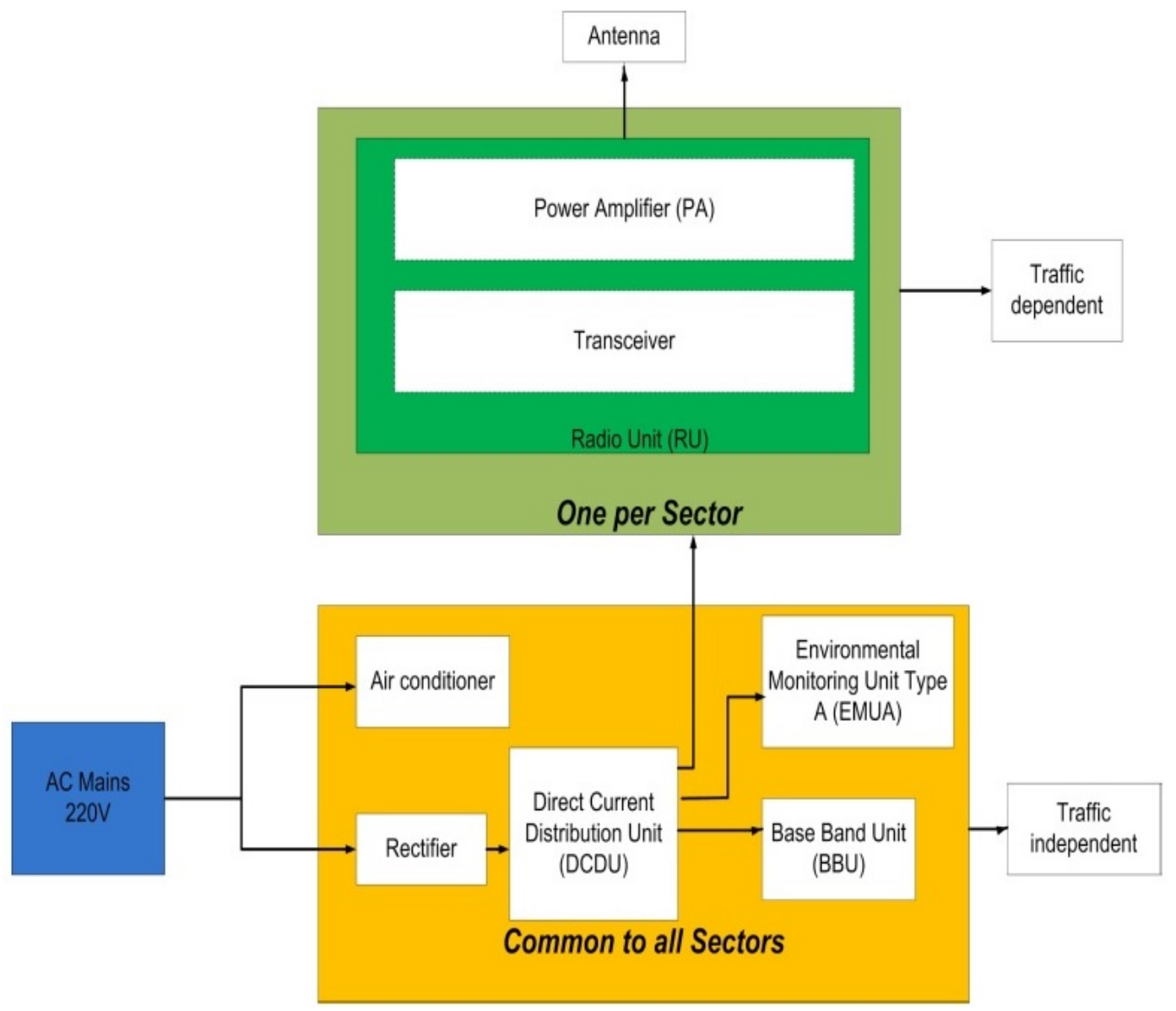

Figure 3.2: Typical Cell Site Components of a BTS [34]. 
which can be generalized to any type of BTSs that are being deployed irrespective of the technology being used. $\mathrm{BB}, \mathrm{RF}$ and $\mathrm{PA}$ have separate sections for receiving (uplink) and transmitting (downlink) purpose.

Total power consumed by BTS depends on the sum of power consumed by each component individually. However, different models have been proposed in [36], [37], [38], [39], and [40] to quantify the total BTS power consumption. Most studies have proposed the linear relationship between transmit power and total power consumed by the BTS. Further, the total power consumed by the BTS is simply the multiple of how many TRXs are being used in a BTS site.

For this study, we refer [40] to model the overall power consumption of a macro BTS stated below as,

$$
P(t)=\left[(1-q) L_{\mathrm{f}}(t) P_{O P}+q P_{O P}\right] \times S_{O N},
$$

where,

$P(t)$ is the power consumed by the BTS at time $t$,

$0 \leq q \leq 1$, determines the level of dependency of $P(t)$ on $L_{\mathrm{f}}$,

$0 \leq L_{\mathrm{f}} \leq 1$, is the Load factor (LF) of cell/sector, LF is defined as the ratio of the number of channels/resource blocks (RBs) in use to the total channels/RBs available in active sector(s), $S_{O N}$ is the total number of active sectors/cells,

$P_{\mathrm{OP}}$ is the maximum operating power per sector/cell when it is fully utilized, i.e., LF $=1$, given by the linear relationship as,

$$
P_{\mathrm{OP}}=g P_{\mathrm{TX}}+h
$$

where,

$P_{T X}$ is the maximum transmit power of a sector/cell.

The values $g$ and $h$ in linear relationship are constants where $g$ is used to dimension the $P_{O P}$ with $P_{T X}$ whereas $h$ dimensions the part of $P_{O P}$ that remains constant irrespective of the 
transmitted power $P_{T X}$.

Hence, in the above illustrated model for power consumption, we only need to calculate the load factor of the cells/sectors to find the power consumed by the BTS at time $t$. While calculating $P_{O P}$, the values used in [40] itself are used. As discussed in [38] and [39], the values have been chosen by analysing the data sheets of existing BTS components in GSM and UMTS network like cooling equipment, power amplifier, antenna interface, RF transceiver, baseband interface, etc. Thus, the chosen values of $g=22.45$ and $h=412.47$ Watts give the maximum total operational power consumed by BTS for a maximum transmit power of 40 Watts $(46 \mathrm{dBm})$ as 1316.4 Watts. 


\section{Chapter 4}

\section{Proposed Markov Decision Process}

As discussed in previous sections, MDP is used as a tool to identify the optimality of the proposed energy saving approach in a cellular communication network. MDP is a dynamic optimization tool that provides a mathematical framework to model a system where the system rewards are partly stochastic and partly under the control of a decision maker. The cellular communication system itself is a stochastic process as user arrival and departure are random in nature, and in order to model such a system, it is necessary to incorporate both the random and static attributes of the system. Hence, finite MDP (FMDP) has been chosen as an appropriate tool that captures such dynamics of the system. MDP is generally defined by the tuple that consists of states, actions, state transition probability, rewards, time epcoh, and discount factor. These basic elements of MDP are used in MDP algorithms to find an optimal policy which maps the actions against states depending on the transition probability and associated rewards.

\subsection{Defining MDP States}

The region under the coverage area of a BTS can be modelled as a Birth-Death process using Markov Chain (MC) where call arrival is modelled by Poisson distribution and the departure time is exponentially distributed. Each birth represents the arrival of the user, 
and each death represents the release of the user after completion of the service. Each state of this $\mathrm{MC}$ is the representation of the number of occupied channels in the region, that is degree of traffic demand. The birth-death process is as shown in Fig. 4.1 below for a six sectored BTS site each containing a maximum of $6 c$ channels.

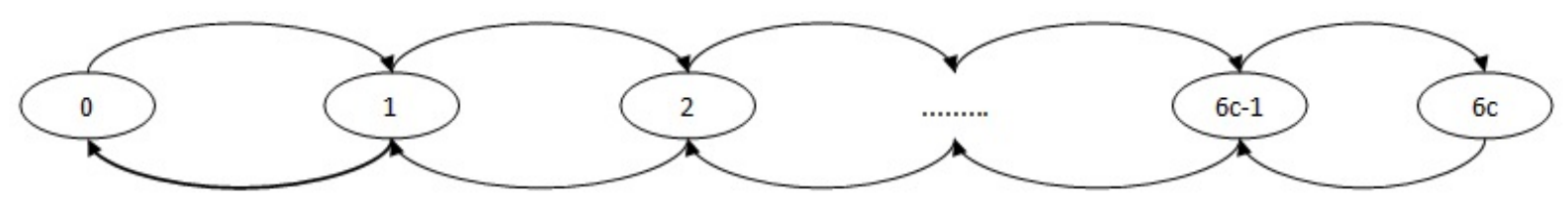

Figure 4.1: Birth Death Process

Hence, for each channel occupancy as an individual state, the first state of the birthdeath process will be 0 and the final state will be $6 c$, i.e., the total channels available in six cells/sectors. The total number of possible states is thus $6 c+1$.

As stated above, each state in the MC represents the number of occupied channels. The policy is developed to save energy in the long term such that the BTS is switched into different modes depending on the present state, i.e., number of channels occupied in the system, which is one of the important inputs to the MDP policy. As there are $6 c+1$ states as shown in Fig. 4.1 that need to be mapped with the number of allowable actions, the decision maker has to handle every traffic transition, which means lot of processing overheads if $c$ is significantly high. This will give more accuracy to the desired outcome but will come at the cost of higher computational complexity. In the proposed MDP, the complexity increases with the increased number of states and actions. The transition probability matrix is the transition probability between the proposed states, which keeps on getting higher as the number of the states increases along with the actions associated with those states. Hence, it is a wiser alternative to reduce the number of the proposed states albeit at the cost of losing some accuracy.

As discussed above, the number of states can be reduced by defining the states as the bounded range of the number of channels being occupied. By this way, the number of the states will reduce considerably depending on how many groups for channel states are 
defined. Such state granulation can be done by setting appropriate threshold values for channel occupancy. This grouping of the range of traffic demand makes the job of central decision maker easier as it eliminates the need to handle every traffic transitions. Let $N_{\text {group }}$ be the total number of states, i.e., groups of channel occupancy being defined. Through this way, the traffic demand is now grouped into $N_{\text {group }}$ number of MDP states. States of the BTS can then be defined as $s \in\left\{1,2, \ldots, N_{\text {group }}\right\}$. The state of the BTS can be determined as,

$$
s=\arg _{i}\left\{c=0, g_{\mathrm{i}-1}<c \leq g_{\mathrm{i}}\right\}, \quad 1<i \leq N_{\text {group }},
$$

where,

$c=$ traffic demand of the BTS,

$g_{\mathrm{i}}=$ maximum channel available in the grouped channel state,

$N_{\text {group }}=$ total number of grouped states.

The maximum channel available for the grouped channel states can be represented as,

$$
g_{\mathrm{s}}=\left[\begin{array}{llll}
g_{1} & g_{2} & \ldots & g_{N_{\text {group }}}
\end{array}\right]
$$

where,

$g_{\mathrm{s}}=$ maximum channel available for the $s^{\text {th }}$ grouped state.

Hence, the state can be granulated by choosing the appropriate value for $g_{\mathrm{s}}$ and $N_{\text {group }}$. Therefore, depending on the MDP policy, the mode of the system has to be changed accordingly to satisfy the traffic demand of the state as well as ensure the system is working in an energy efficient manner. This means, more sectors has to be opened when the traffic demand increases and vice versa when traffic demand decreases, under the optimal policy. This is discussed further in Section 4.2. 


\subsection{Possible MDP Actions}

As the user moves in and out of the process, the state of the process keeps on changing. However, the controller takes actions only at specific time intervals defined by the epoch time. The following actions are proposed which can be taken depending on the varying degree of traffic demand along with the optimal policy in place as,

$$
a_{\mathrm{s}, \mathrm{t}} \in A=\left\{\begin{array}{lr}
1, & \text { the BTS switches to adjacent upper mode } \\
-1, & \text { the BTS switches to adjacent lower mode } \\
0, & \text { the BTS stays in current mode, }
\end{array}\right.
$$

where,

$\mathrm{a}_{\mathrm{s}, \mathrm{t}}=$ action taken in state $s$ at time $t$.

When,

$\mathrm{a}_{\mathrm{s}, \mathrm{t}}=1$, the BTS reconfigures to adjacent upper mode, e.g. if it is currently in two-sector mode, it moves to three-sector mode.

$\mathrm{a}_{\mathrm{s}, \mathrm{t}}=-1$, the BTS reconfigures to the adjacent lower mode, e.g. if it is currently in threesector mode, it moves to two-sector mode.

$\mathrm{a}_{\mathrm{s}, \mathrm{t}}=0$, the process stays in the same mode it is currently in.

Only one action is allowed at one instance. Likewise, all actions are not allowed at all states. All the possible mode switching decisions to adjacent modes as stated above can be taken at all times except as mentioned below.

- $\mathrm{a}_{\mathrm{s}, \mathrm{t}}=-1$ is not allowed when BTS is in Mode 1, i.e., sleep mode.

- $\mathrm{a}_{\mathrm{s}, \mathrm{t}}=1$ is not allowed when BTS is in Mode 5, i.e., six-sector mode.

Only adjacent modes are allowed to be activated considering the physical limitation of BTS transition time for some configuration of BTS deployment and the traffic load handling capacity of each modes. It is expected that the traffic requirement does not vary significantly in a short duration. 


\subsection{Analysis of MDP Decision Epoch}

DTMDP are characterised by taking decision at discrete time intervals unlike continuous time MDPs where decision maker can choose to take decisions whenever they want. Such pre-defined discrete time interval is used to inspect the state of the process and take the appropriate action available for that state. That discrete time interval is known as MDP decision epoch, denoted by $\delta$. It is a critical decision for the central decision maker to choose the appropriate decision epoch time $\delta$ as that directly affects the performance of the whole optimization process. There are pros and cons of selecting the too short or too long $\delta$, which will have a significant impact on the expected energy saving of the system.

Too short $\delta$ will react very fast in line with the fast varying traffic and is expected to save more energy at least in theoretical sense as it optimises itself instantly according to the traffic demand. However, such reaction will be limited by the network's capacity to respond to such reaction as network components will require some time to activate and deactivate as and when necessary. Too short $\delta$ also creates ping pong effect as system might move between modes too frequently, thereby undermining the efficacy of the system.

Likewise, too long decision epoch will have a bearing on the reaction time of the network to time varying traffic demand. It is expected that the energy saving efficiency is directly proportional to the faster reaction time, hence the longer decision epoch will severely hamper that effectiveness of the system under consideration. Hence, there is a trade-off between higher or lower time epoch and energy efficiency of the system.

\subsection{Transition Probability Matrix Between States}

As already discussed, this energy minimization problem is formulated as a MDP problem. Due to the probabilistic nature of user arrival and departure, the Markov process also transits randomly from one state to another in any pre-defined time interval, $\delta$, as,

$$
P_{\delta}^{\mathrm{a}}\left(s, s^{\prime}\right)=P\left(s(t+\delta)=s^{\prime} \mid s(t)=s, a(t)=a\right),
$$


which shows the probability that the action $a$ taken in state $s$ at time $t$ will transit the MDP system state to $s^{\prime}$ at next time step $t+\delta$ where $\lambda \delta \ll 1$ and $\mu \delta \ll 1$ where $\lambda$ and $\mu$ are arrival and departure rate respectively.

This Markov process can be visualised in a different way by viewing at certain time intervals separated by the time interval $\delta$, also known as decision epoch in this study as discussed in Section 4.3. Referring to [35], such visualization is illustrated in detail in the following statements. For this paragraph only, each state increment represents an increase of one channel occupancy, different from the rest of this study. The Poisson process can be approximated by Bernoulli processes where the transition probability from state $s$ to state $s^{\prime}$ in the sampled time chain can be defined as $q_{s s^{\prime}} \delta$ for all $s \neq s^{\prime}$. Hence, the transition probability out of state $s$ to state $s^{\prime}$ is given by $\lambda \delta$ for arrival and from state $s$ to state $s^{\prime}$ by $n \mu \delta$ for departure where $n$ is the number of occupied channels in state $s^{\prime}$. Since the transition probabilities in and out of the state decreases with decreasing $\delta$, there is a high chance that there will be no transition out of the state in the said time increment $\delta$. This is modelled by self-transition, i.e., one minus the probability of transitions out of the state as shown in Fig. 4.2. Likewise, arrival rate depends on the state of the neighbouring BTS as well. It is the aggregate of new call arrivals as well as handover calls from the neighbouring BTSs. For ease of study, summation of both new call arrival rate and handover call rate is taken as $\lambda$. Departure rate also consists of both terminated calls as well as calls handed over to another BTS.

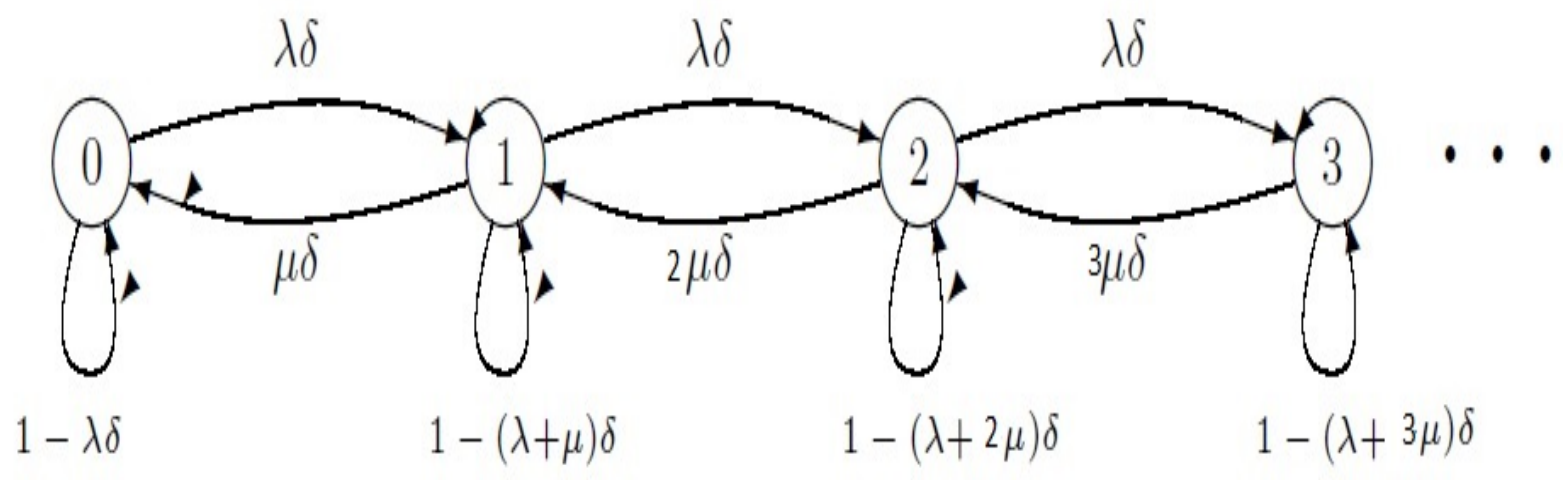

Figure 4.2: Sampled Time Approximation to Markov Process 
In this study, as discussed in Section 4.1, the smaller states, i.e., each channel occupancy representing one state, in a birth-death process are grouped together to form a state that is grouped in pre-determined boundary value for the group. This way, the number of states in a process are reduced, thereby reducing the complexity of the system when choosing the optimal MDP policy. As such, the transition probability matrix of the process that was defined earlier has to be changed accordingly to represent the transition probability between the newly defined grouped states.

As discussed earlier, for the grouped states, the state transition probability matrix (TPM), i.e., $P_{\left(s, s^{\prime}\right)}$ for all actions $\left(\forall a_{s, t} \in A\right)$ can now be stated as,

$$
P\left(s, s^{\prime}\right)=\left\{\begin{array}{lr}
\operatorname{Pr} \cdot\left[c=g_{\mathrm{s}} \mid g_{\mathrm{s}-1}<c \leq g_{\mathrm{s}}\right] \lambda \delta, & 1<\left(s^{\prime}=s+1\right) \leq N_{\text {group }}, \\
\operatorname{Pr} \cdot\left[c=g_{\mathrm{s}-1}+1 \mid g_{\mathrm{s}-1}<c \leq g_{\mathrm{s}}\right]\left(g_{\mathrm{s}-1}+1\right) \mu \delta, & 1 \leq\left(s^{\prime}=s-1\right)<N_{\text {group }}, \\
1-P(s, s+1)-P(s, s-1), & s^{\prime}=s \\
0, & \text { otherwise }
\end{array}\right.
$$

where,

$c=$ number of channels occupied (traffic demand),

$g_{\mathrm{s}}=$ boundary number of the $s^{\text {th }}$ grouped state,

$\delta=$ time epoch,

$\lambda=$ arrival rate,

$\mu=$ departure rate.

For $s^{\prime}=s+1$, i.e., the movement of state from lower to upper adjacent state, the transition probability is the conditional probability multiplied with the transition rate, i.e., $\lambda \delta$ from lower state to upper state. Conditional probability in this case is the probability of channel occupancy change from lower group to adjacent upper group.

Likewise, for $s^{\prime}=s-1$, i.e., the movement of state from upper to lower adjacent state, the transition probability is the conditional probability multiplied with the transition rate, 
i.e., $\mu \delta\left(g_{s-1}+1\right)$ from upper state to lower state. Conditional probability in this case is the probability of channel occupancy change from upper group to adjacent lower group.

Similarly, for $s^{\prime}=s$, i.e., remaining in the same state, transition probability is one minus the transition probability of moving into and out of the state.

For this study, the process has been granulated into five groups, i.e., $N_{\text {group }}=5$, and

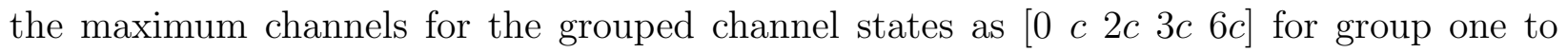
five respectively, where $c$ is the number of channels available per sector. This boundary number has been set to match with the number of channels available when the number of sectors are activated in different modes, e.g. $3 c$ channels are available when three sectors are activated. The Markov process can then be illustrated as shown in Fig. 4.3 for the given values. The arrows moving from lower grouped states to upper grouped states represent the transition probability of moving from lower channel occupancy to higher channel occupancy state. Likewise, the arrows moving from upper grouped states to lower grouped states represent the transition probability of moving from upper channel occupancy to lower channel occupancy. The long arrows starting and ending in the same grouped state represent the transition probability of remaining in the same state.

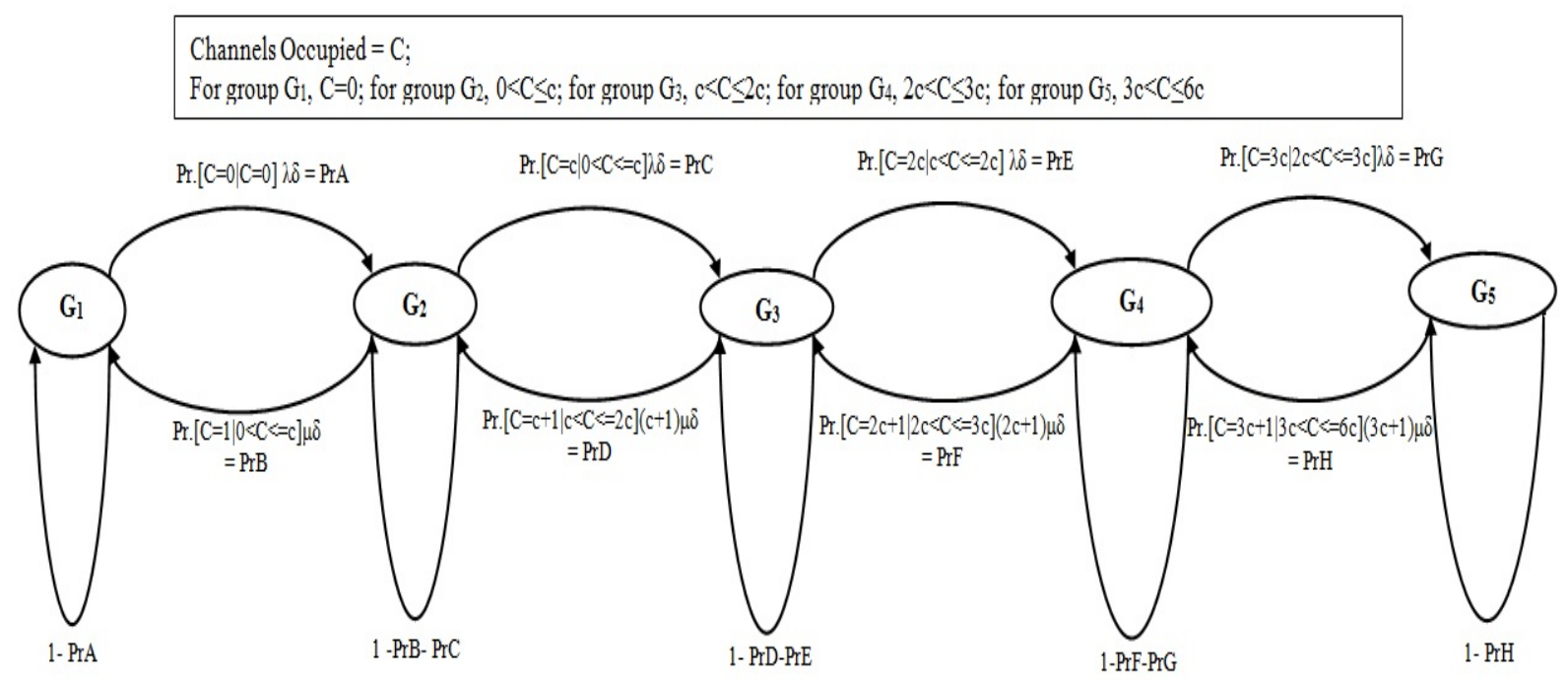

Figure 4.3: Grouped Transition Probability Matrix of a Markov Process 


\subsection{Reward Function Analysis}

Rewards in this study is the expected energy saving when certain action is taken in a state to activate the required BTS mode of operation. The power consumption while dealing with rewards is the instantaneous power consumed by the base station which is modelled as per the power consumption model stated in (3.2). The actual reward in this study is the power savings due to the action taken compared to the baseline mode of power consumption. The baseline power consumption mode is defined as the mode when BTS is operating in six sector mode irrespective of the instantaneous traffic intensity during the day, i.e., $S_{O N}=6$, as shown below,

$$
P_{\text {baseline }(6 \mathrm{~S})}(t)=\left[(1-q) L_{\mathrm{f}}(t) P_{\mathrm{OP}}+q P_{\mathrm{OP}}\right] \times 6 \text {. }
$$

Likewise, the power consumed when action $a$ is taken to activate specific configuration of mode is given as,

$$
P_{\text {mode }_{a}}(t)=\left[(1-q) L_{\mathrm{f}}(t) P_{\mathrm{OP}}+q P_{\mathrm{OP}}\right] \times \operatorname{mode}_{a}(t)
$$

where,

$\operatorname{mode}_{a}(t) \in[0,1,2,3,6]$, which indicates number of sectors $\mathrm{ON}$ at $t$, which is given by the result from $\operatorname{mode}_{a}(\mathrm{t}-\delta)$ and action $a$.

Hence, such reward is the instantaneous power consumption difference between the baseline mode and the mode that is activated after the action is taken, i.e., power saving. Let us denote it by $P_{\text {save }\left(s^{\prime}, a\right)}$ which can be expressed as,

$$
P_{\text {save }\left(s^{\prime}, a\right)}=P_{\text {baseline }(6 \mathrm{~S})}(t)-P_{\text {mode }_{\mathrm{a}}}(t)
$$

where,

$P_{\text {baseline(6S) }}(t)$ is the baseline power consumption when six sector mode is activated,

$P_{\text {mode }_{\mathrm{a}}}$ is the power consumption at specific mode activated by taking action $a$, 
Likewise, for sleep mode, each sector of BTS is assumed to consume a fixed power of 12 Watts, i.e., $P_{\text {sleep }}(t)=12 \times 6=72$ Watts.

The above mentioned rewards are the maximum expected rewards that can be obtained after an action is taken. However, there are other factors, specifically transition cost and delay cost in this study, that have to be considered as well so as to obtain the actual rewards. Transition cost is the cost incurred only when mode switching takes place, i.e., BTS mode changes from one mode to another when $a_{\mathrm{s}, \mathrm{t}}=\{1,-1\}$, irrespective of moving to upper mode or lower mode. This cost can be taken as a lump sum cost incurred specially due to user handover, hardware initialization, processing time, and other factors. Another important cost, i.e., delay cost, is the cost incurred when the incoming call request has to wait before being served due to unavailability of channels. It has been observed that the MDP process chooses to remain in lower mode rather than moving to upper mode, because the reward is higher in lower modes in spite of more channels being available in upper modes. This might compromise the quality of the service of the system operation. Hence, this cost has been introduced to ensure the system is made to serve the incoming call request without much delay by opening the highest mode, i.e., higher number of channels, available in the system. Significance of the delay cost is observed when the system takes action to remain in the same mode of operation, i.e., $a_{\mathrm{s}, \mathrm{t}}=0$, in spite of the system being in a state to address that call request by configuring itself into the next higher mode. The actual reward can then be restated below as,

$$
R\left(s^{\prime}, a\right)=\alpha\left(P_{\text {baseline }(6 \mathrm{~S})}(t)-P_{\text {mode }_{\mathrm{a}}}(t)\right)-\beta\left(\text { Cost }_{\text {transition }}+\text { Cost }_{\text {delay }}\right)
$$

where,

Cost $_{\text {transition }}=0$, for $a_{\mathrm{s}, \mathrm{t}}=0$,

Cost $_{\text {delay }}=0$, for $a_{\mathrm{s}, \mathrm{t}}=\{1,-1\}$,

$\alpha, \beta=$ coefficients for power to reward and cost to reward respectively.

Inclusion of these costs in a MDP policy development have unique effects on the overall energy efficient operation of the system. Delay cost always decreases the total rewards 
obtained when the system chooses to stay in the same mode in spite of the number of delayed call requests. This means rewards will be higher if the system chooses to open more sectors, especially to higher modes, as there is demand for more channels at that instance, which is necessary from maintaining the QoS point of view. However, this transition of the system to higher mode consumes high energy. Hence, there is a trade-off between maintaining QoS and consuming less energy.

Likewise, transition cost is necessary to reflect the absolute reward obtained through mode changes. BTS site operation after mode transition might give high reward but the cost the system has to incur for switching between modes actually decreases the real value of the reward. Hence, the inclusion of the transition cost in determining the actual reward makes the system take full account of the cost the system has to incur when switching from one mode to other, irrespective of it is higher mode or lower mode thereby avoiding frequent mode changes. Further, effect of this cost in the overall energy consumption depends on either the system is supposed to move to upper mode or lower mode. If it was supposed to move to lower mode, then the system will consume more energy due to transition cost. However, high power saving will be observed if it was supposed to move to upper mode, but stays at lower mode due to this cost. Therefore, the net effect of this cost will depend on how often the system chooses to stay in the same state, even though it was supposed to switch modes had this cost not been considered.

\subsection{MDP Optimization Problem Analysis}

The MDP optimization problem is solved by finding the optimal policy that maps the actions to states which will give the expected maximum reward in the long term. In this study, the maximum reward is the maximum energy savings when actions are taken. The reward function can thus be defined as the amount of power saving that can be achieved when action $a$ is taken in state $s$ as, 


$$
\mathbf{R}(\mathbf{s}, \mathbf{a})=\sum_{s^{\prime} \in S} P_{\delta}\left(s, s^{\prime}\right) \times R\left(s^{\prime}, a\right),
$$

where $P_{\delta}\left(s, s^{\prime}\right)$ is the transition probability for the MDP epoch time $\delta$ and $R\left(s^{\prime}, a\right)$ is the power savings, i.e., rewards for action a compared to a baseline power consumption system as shown in (4.7). Baseline power consumption means BTS is operating in six sector mode regardless of the variation in traffic intensity during the day. The MDP optimization problem can be stated below as,

$$
\pi(s)=\arg \max _{a} \mathbf{R}(\mathbf{s}, \mathbf{a})
$$

subject to

$$
\begin{aligned}
a_{\mathrm{s}, \mathrm{t}} g_{\mathrm{s}} \leq g_{\mathrm{N}_{\text {group }}}, & \text { for } \mathrm{a}_{\mathrm{s}, \mathrm{t}}=1, \\
-a_{\mathrm{s}, \mathrm{t}} g_{\mathrm{s}} \geq 0, & \text { for } \mathrm{a}_{s, t}=-1 .
\end{aligned}
$$

The solution of this optimization problem is the optimal policy $\pi(\mathrm{s})$, such that action $a$ taken in state $s$ out of all the possible actions, $a_{\mathrm{s}, \mathrm{t}} \in A$, maximizes the total long term reward. Two popular dynamic programming algorithms to tackle this optimization problem are policy iteration and value iteration. The solution from both the algorithm is the optimal MDP policy which gives the maximum expected reward as,

$$
\pi(s)=\arg \max _{a}\left\{\sum_{s^{\prime} \in S} P_{\delta}\left(s, s^{\prime}\right)\left(R\left(s^{\prime}, a\right)+\gamma V\left(s^{\prime}, a\right)\right)\right\}
$$

The policy iteration algorithm as shown in (4.14) exhaustively searches through all the possible set of policies and converges to the optimal solution that gives maximum reward. Likewise, the value iteration algorithm as shown below, 


$$
V(s)=\sum_{s^{\prime} \in S} P_{\delta}\left(s, s^{\prime}\right)\left(R_{\pi(\mathrm{s})}\left(s^{\prime}\right)+\gamma V\left(s^{\prime}\right)\right),
$$

continuously searches for the new policy that maximizes the instantaneous value function $V(s)$ until such maximized value converges to some stationary value. In this study, policy iteration algorithm has been used as this takes many but fewer iterations. Likewise, policy iteration improves the policy at each step and encounters one policy at once and is guaranteed to converge. When this happens, the value function of such policy is optimal value function with optimal policy.

The policy has to be evaluated taking into consideration the reward (power saving) of remaining in the present state and the subsequent reward that results after the state transition. One of the several popular ways of measuring the performance of Markov chains is the long run expected average cost per unit time. In order to evaluate this measure, the steady state probabilities $\pi_{1}, \pi_{2}, \pi_{3}, \pi_{4}$ and $\pi_{5}$ have to be calculated for the Markov chain by solving the steady state equations obtained through transition probability matrix, $P$. This will give us the limiting values of the probability of the system being in each of the states, irrespective of the initial state. It has been shown that the long run expected average cost per unit time converges to the actual average cost per unit time as time goes to infinity when the effect of initial state disappears [32]. 


\section{Chapter 5}

\section{Simulation/Numerical Results and}

\section{Analysis}

This section presents the simulation results of the proposed energy efficient management of BTS using MDP as an optimization tool with practical values. Through extensive simulation, the effectiveness of the proposed method has been verified. Table 5.1 lists the values of different simulation parameters used in obtaining the results in this chapter, unless otherwise mentioned. The chosen values for the variables are the realistic values used in practical environment. Matlab` alongwith 'MDP toolbox version 4.0' [41] were used as simulation tools to obtain the results of the study.

The main aim of this study was to investigate the energy efficiency operation of a BTS by employing MDP as an optimization tool. However, simply employing MDP as an optimization tool does not guarantee the energy optimization operation of a BTS. Various parameters such as number of available channels, traffic arrival rate, service time, transition cost between modes, delay cost of wait time, time epoch etc., and each plays a significant role in the expected operation of a BTS. Appropriately choosing the value of those parameters is quite important, but this also means that the efficiency of the BTS operation can be optimized as per the localized needs of the BTS.

The efficiency of the methodology has been proven through the measurement of power 
Table 5.1: Simulation Parameters

\begin{tabular}{||c|c|c|c||}
\hline Parameters & Definition & Value Used & Unit \\
\hline \hline$c$ & No. of channels per sector & 5 & - \\
\hline$g$ & $\begin{array}{l}\text { Dimensions operating power through } \\
\text { transmit power }\end{array}$ & 22.6 & - \\
\hline$h$ & Constant Power & 412.4 & Watts \\
\hline$q$ & weighting constant & 0.7 & - \\
\hline$P_{\mathrm{TX}}$ & Maximum Transmit Power of a cell/sector & $40(46)$ & Watts $(\mathrm{dBm})$ \\
\hline$P_{\text {Sleep }}$ & Sleep mode power consumption per sector & 12 & Watts \\
\hline$N_{\text {group }}$ & Number of grouped states & 5 & - \\
\hline$\lambda_{-}$max & Maximum call arrival rate & 1000 & calls $/$ hour \\
\hline$h_{\mathrm{t}}$ & Average call holding time & 72 & seconds \\
\hline$\mu$ & Service rate & $1 / h_{\mathrm{t}}$ & calls $/$ second \\
\hline$\alpha$ & trade-off value & 1 & - \\
\hline$\beta$ & trade-off value & 1 & - \\
\hline$\gamma$ & Discount factor & 0.9 & - \\
\hline
\end{tabular}

consumption with and without MDP. Impact of the parameters, such as, call arrival rate, epoch time, transition cost, delay cost, etc. on action distribution and mode distribution are investigated. Epoch time's effect on power saving is analysed. Further, the results also vary when time homogeneous, i.e., same traffic load throughout the day, and time inhomogeneous traffic is considered.

As time homogeneous traffic is unrealistic in practical situation and are limited to theoretical interest, only time inhomogeneous traffic are considered in this study, which is the actual traffic in daily environment. For that purpose, the normalized traffic profile as shown in Fig. 5.1 is used. The figure shows the typical traffic profile of a week day. It should be noted that the traffic profile is granulated for every half an hour interval. 


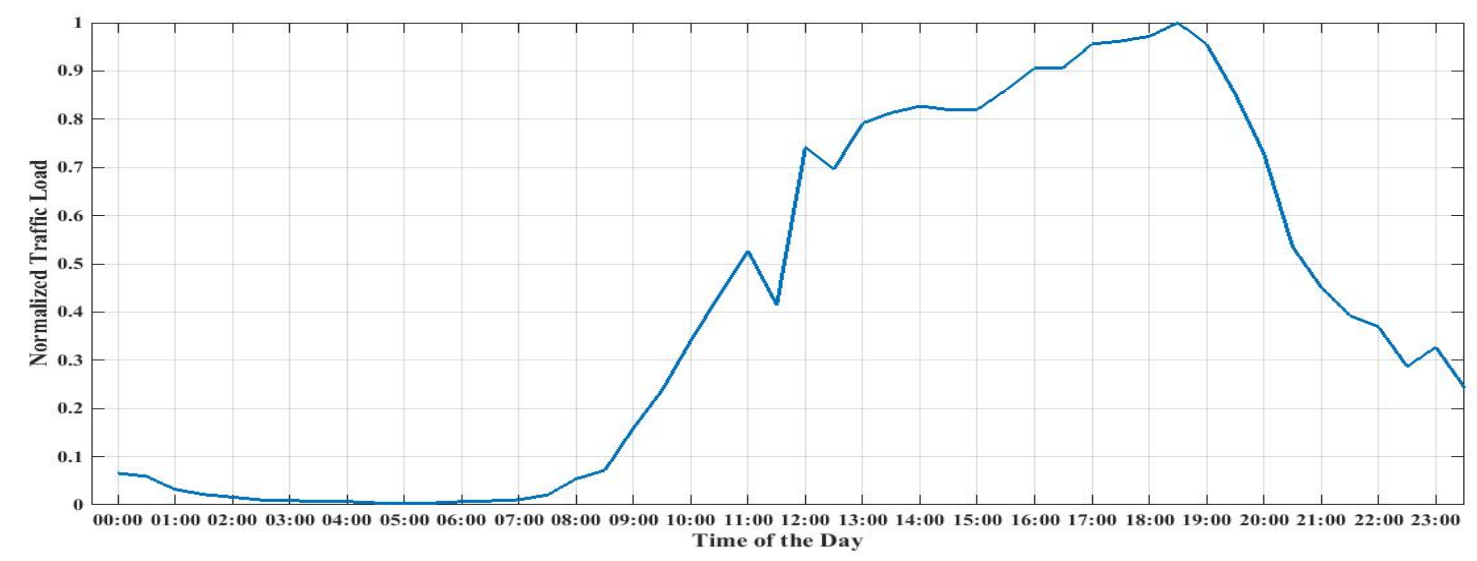

Figure 5.1: Normalized Traffic Load of a Typical Weekday. [33]

The maximum call arrival rate $\left(\lambda \_\max \right)$ during the day is taken as 1000 calls per hour with the variation being dictated by the normalized traffic profile at other times. This is done to ensure that the proposed methodology works well under the real traffic variation at different times of the day. Epoch time is taken as one second unless mentioned otherwise.

\subsection{Power Consumption and Saving Analysis}

\subsubsection{Power Consumption and Saving Analysis Without Delay Cost and Transition Cost}

As stated throughout the study, the proposed methodology in minimizing power consumption of a BTS using MDP is verified through Fig. 5.2. It can be seen that power consumption with MDP is significantly lower than that without MDP while both transition and delay cost are set to zero. The power consumption also follows the traffic pattern as shown in Fig. 5.1. It should be noted that the power consumption at particular time of the day is the cumulative power consumed by the BTS in half-hour period which matches with the traffic profile used in this study. As stated above, the traffic profile changes at every half an hour interval. 


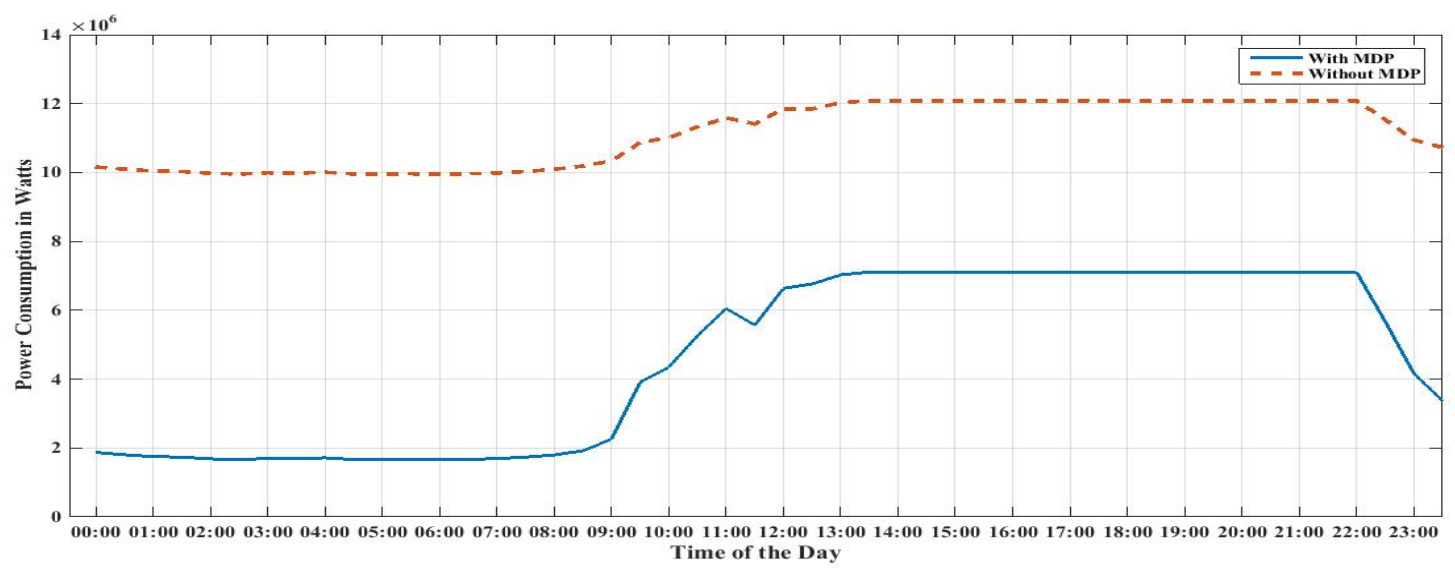

Figure 5.2: Daily Power Consumption With and Without MDP. Max $\lambda=1000$, Transition Cost $=0$, Delay Cost $=0$, Epoch $=1 \mathrm{~s}$

Fig. 5.3 shows the actual percentage of power saved during the day. Power saving depends on how accurately the MDP responds to the change in traffic load. Maximum power savings of $83 \%$ and minimum power saving of $41 \%$ is observed during period of low traffic time and high traffic time respectively. However, daily average power saving is observed at around $60 \%$. As can be seen in the figure, the saving is high at around early hours of the morning which is due to the fact that this is the region of lowest traffic or no traffic at all. This means, there is high probability of power saving at low traffic times because the system is operating with few sectors $\mathrm{ON}$ at that time. As the traffic grows, the power saving decreases as the system operates with more number of sectors ON. 


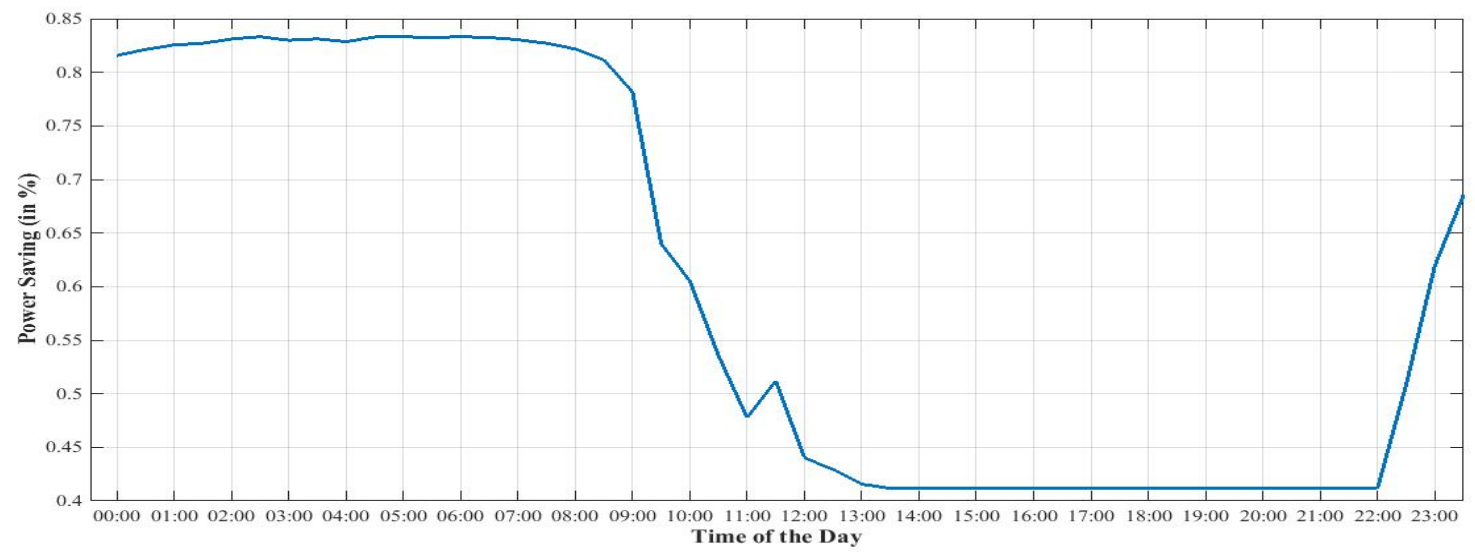

Figure 5.3: Daily Power Saving. Max $\lambda=1000$, Transition Cost $=0$, Delay Cost $=0$, Epoch $=1 \mathrm{~s}$

\subsubsection{Power Consumption and Saving Analysis With Transition Cost}

Fig. 5.4 shows the power consumption of a BTS when the effect of transition cost is considered. It can be seen that the power consumption with MDP has increased significantly at around $80 \%$ in the period of low traffic, but remaining more or less same in high traffic period. It is because the BTS now tends to stay on the same mode rather than switching modes frequently as mode switching will incur the cost, most probably in higher mode if power consumption is increased, which affects the long term energy saving that can be received from the system. The power consumption at particular time of the day is the cumulative power consumed by the BTS in a half-hour period. 


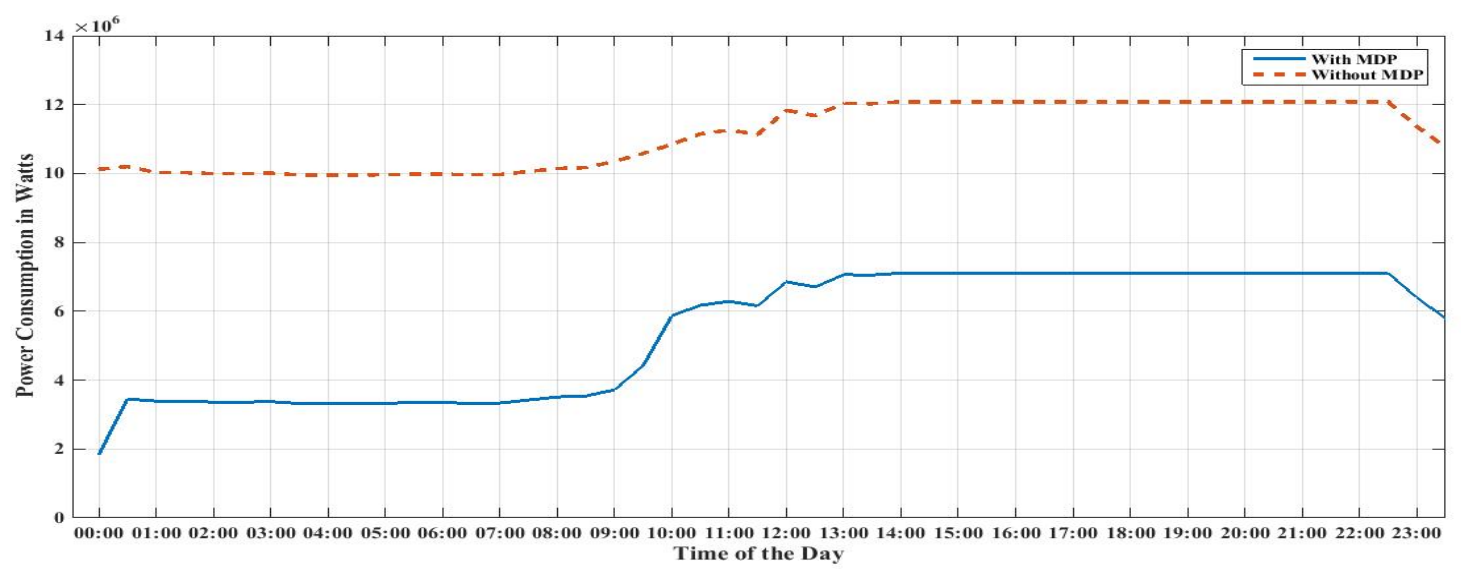

Figure 5.4: Daily Power Consumption Analysis With and Without MDP. Max $\lambda=1000$, Transition Cost $=1000$, Delay Cost $=0$, Epoch $=1 \mathrm{~s}$

Power saving when BTS is operated by introducing the transition cost is shown in Fig. 5.5. The power saving pattern looks similar to the one in Fig. 5.3 when the system is operated without transition and delay cost, but the power saving is significantly reduced during low traffic times at around $66 \%$ when the system is operating with transition cost. During high traffic arrival time, the power saving is similar to the one without transition and delay cost.

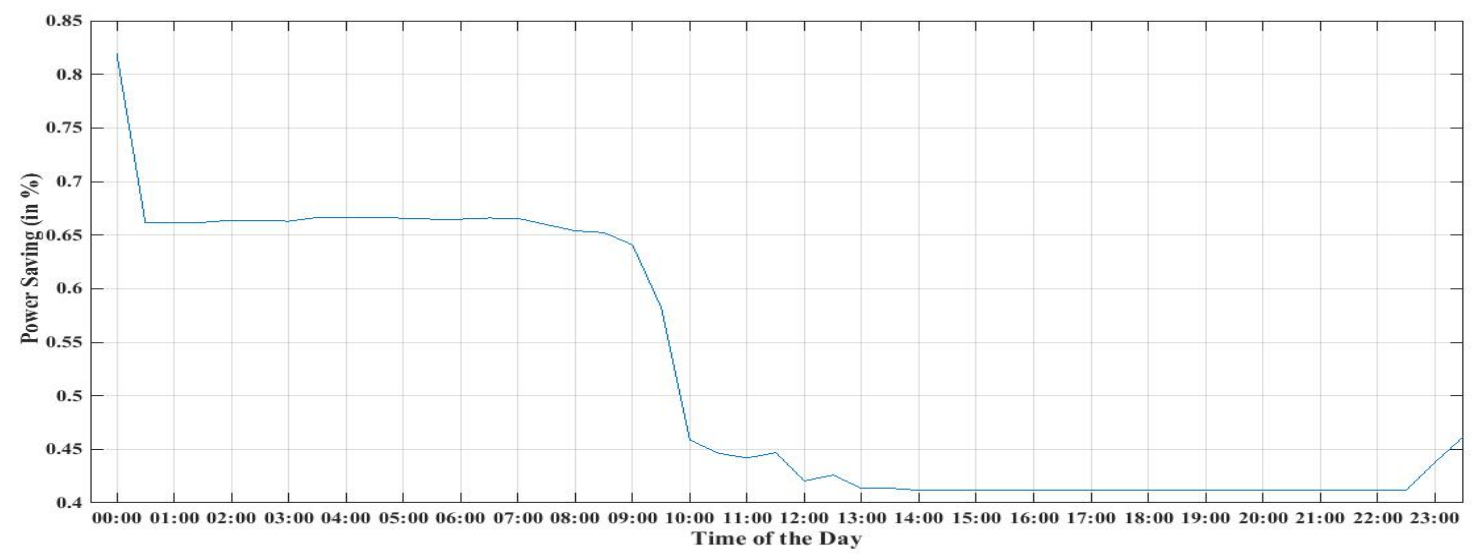

Figure 5.5: Daily Power Saving With and Without MDP. Max $\lambda=1000$, Transition Cost $=$ 1000 , Delay Cost $=0$, Epoch $=1 \mathrm{~s}$ 


\subsubsection{Power Consumption and Saving Analysis With Delay Cost}

Fig. 5.6 shows the power consumption of a BTS when delay cost is introduced. Delay cost is the cost incurred by the system when it is unable to render service to the call request as there are no channels available in the system. This is the special case when the system chooses to stay in the lower mode even when the channels will be available had it moved to the next available upper mode. This cost ensures that the system will look to serve the incoming call by moving to next upper mode rather than staying in the same mode, when it was supposed to get higher energy saving to do so, but not so with delay cost's effect in calculating the reward. The power consumption at particular time of the day is the cumulative power consumed by the BTS in a half-hour period.

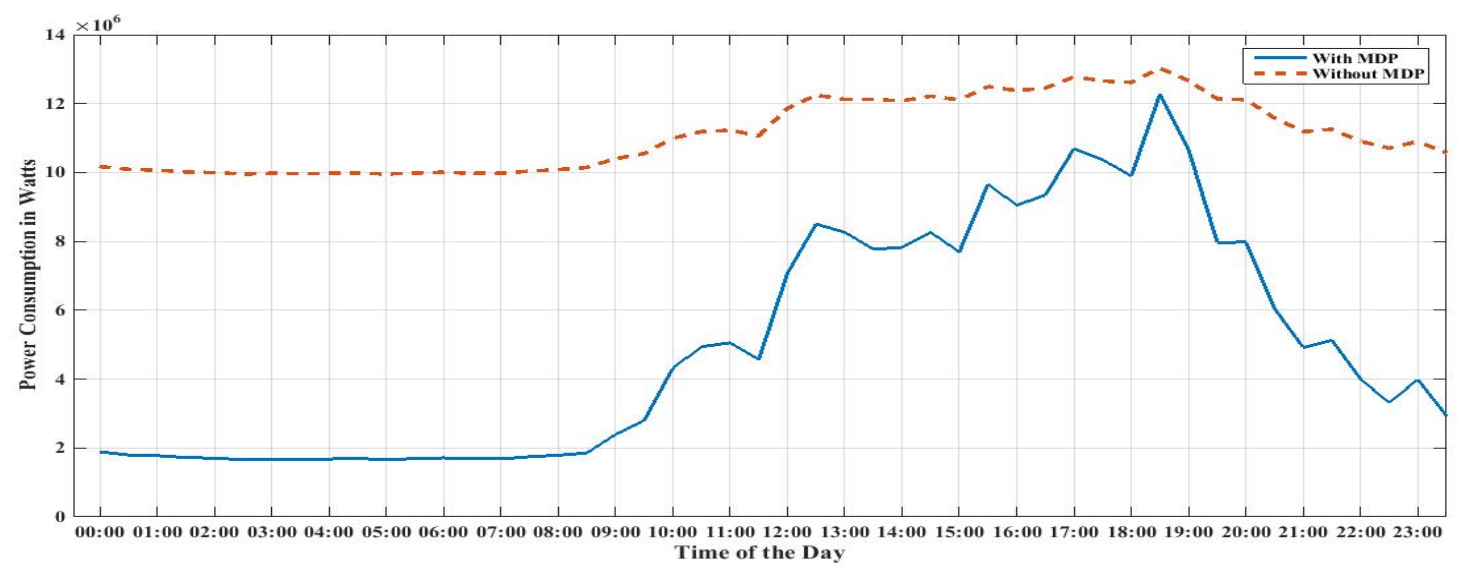

Figure 5.6: Daily Power Consumption Analysis With and Without MDP. Max $\lambda=1000$, Transition Cost $=0$, Delay Cost $=100$, Epoch $=1$ s

It can be seen that at some point the system is consuming almost same power regardless of the system is running with MDP or without MDP because the system is operating at highest mode to serve high traffic load. Introduction of delay cost forces the system to operate in 6-sector mode when the traffic load is high. This figure can be benchmark to understand if the channels available in the system is enough to support the high traffic load. As can be seen from the figure, when the traffic load is high, the power consumption with and 
without MDP are almost similar. That means the system is operating in 6-sector mode quite often. Equal power consumption for the system with and without MDP means the system is operating at its full potential and there might be instances when call has been dropped. When the traffic load is high, there is no difference in power saving with or without MDP as the system operates in 6-sector mode. It is further discussed in detail in Section 5.2.3.

Fig. 5.7 shows the power saving when delay cost is introduced. This can be clearly seen in the figure that power saving nearly approaches zero during high traffic arrival time. This is because as the system keeps operating more in 6-sector mode, less power is being saved.

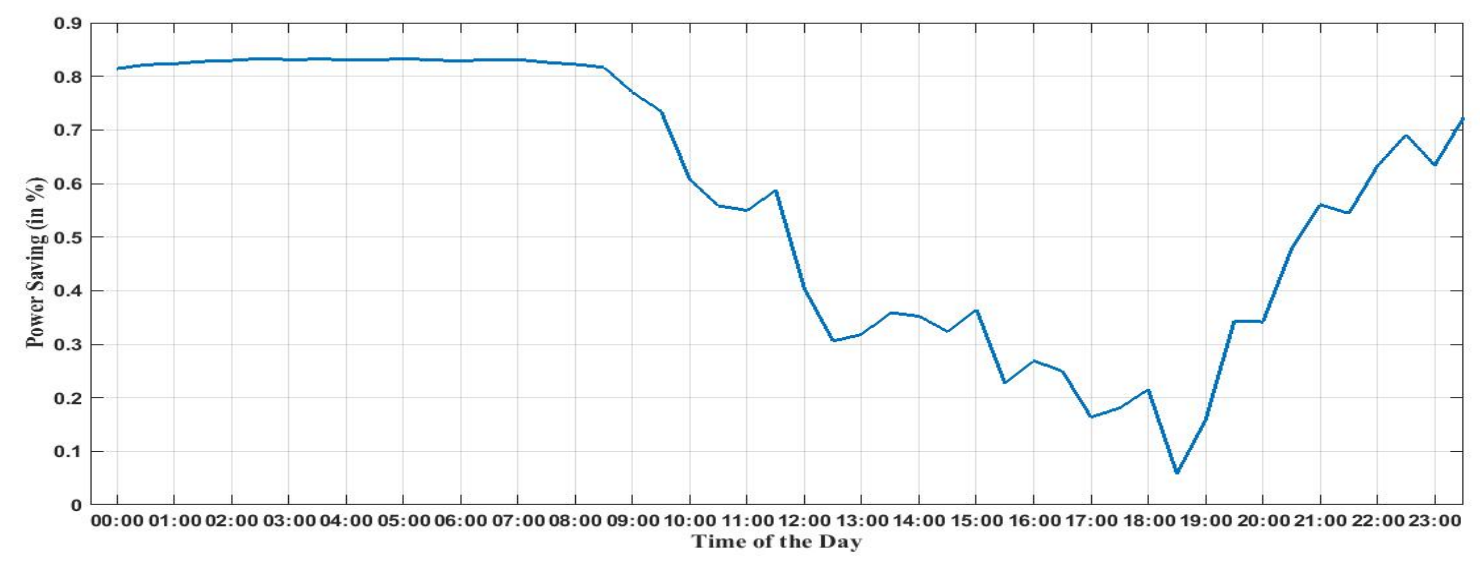

Figure 5.7: Daily Power Saving. Max $\lambda=1000$, Transition Cost $=0$, Delay Cost $=100$, Epoch $=1 \mathrm{~s}$

\subsection{BTS Mode Distribution Analysis}

\subsubsection{Mode Distribution Analysis Without Delay and Transition Cost}

Fig. 5.8 shows the mode distribution of BTS during a typical day when MDP optimization tool is employed. It can be seen that the BTS generally do not operate in sleep mode and rarely goes to 6 -sector mode when it is operated without delay and transition cost. Hence, 
the mode occupancy distribution is shared between omni, 2-Sector and 3-Sector modes.

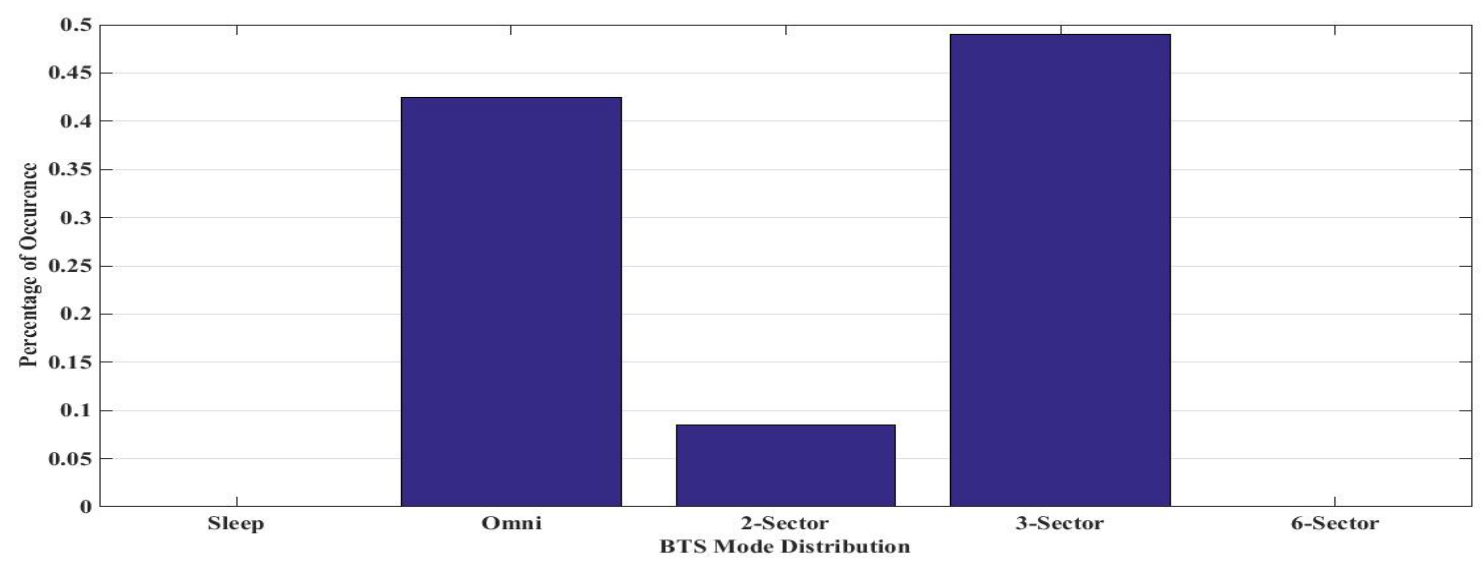

Figure 5.8: BTS Mode Distribution Analysis. Max $\lambda=1000$, Transition Cost $=0$, Delay Cost $=0$, Epoch $=1 \mathrm{~s}$

\subsubsection{Mode Distribution Analysis With Transition Cost}

When transition cost is introduced, the system operation is affected as it tends to stay in the same modes rather than switch modes to move to another mode as shown in Fig. 5.9. As shown in Fig. 5.9. the system is in either 2-sector or 3-sector mode for the majority of the time. 


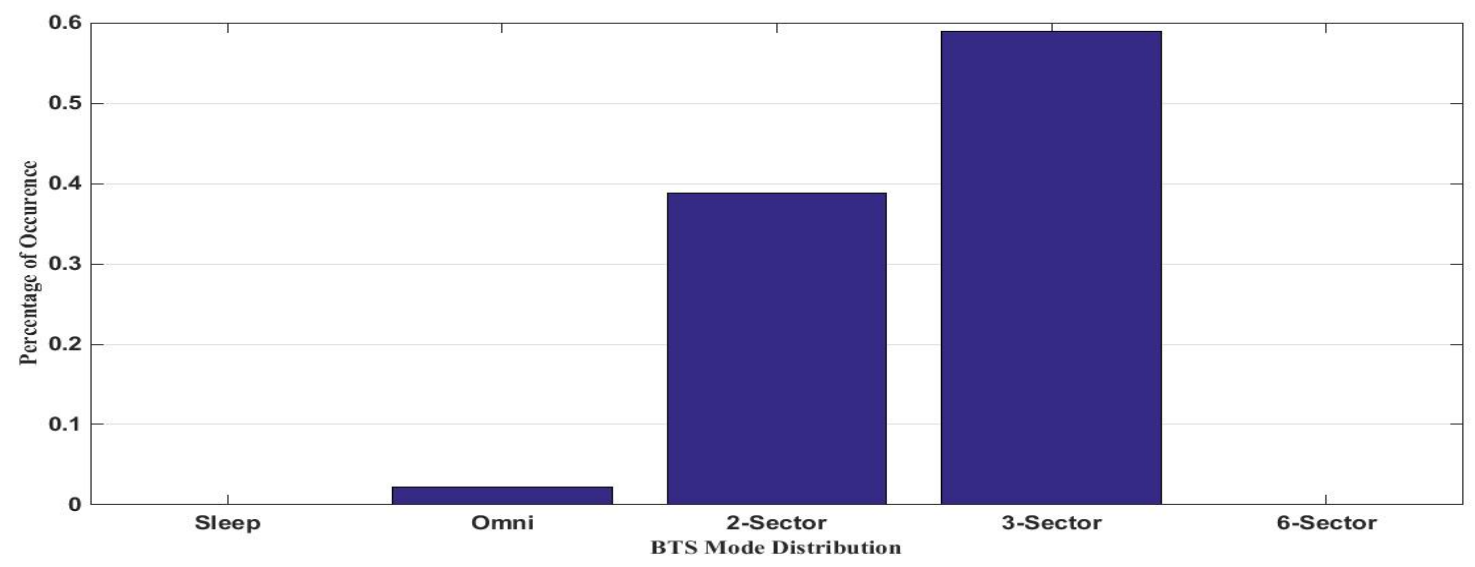

Figure 5.9: BTS Mode Distribution Analysis. Max $\lambda=1000$, Transition Cost $=1000$, Delay Cost $=0$, Epoch $=1 \mathrm{~s}$

\subsubsection{Mode Distribution Analysis With Delay Cost}

Fig. 5.10 shows the BTS mode distribution when delay cost is introduced. As seen in sections above, mode occupancy ratio was prominently distributed between omni, 2-sector and 3-sector mode. However, after the introduction of delay cost, i.e., the cost incurred by the system when making the call request wait, the mode occupancy distribution has moved to 6-Sector configuration as well. This cost affects the performance of the system by taking into consideration the degradation of the quality of the service when call request has to be made to wait, which is reflected as delay cost. 


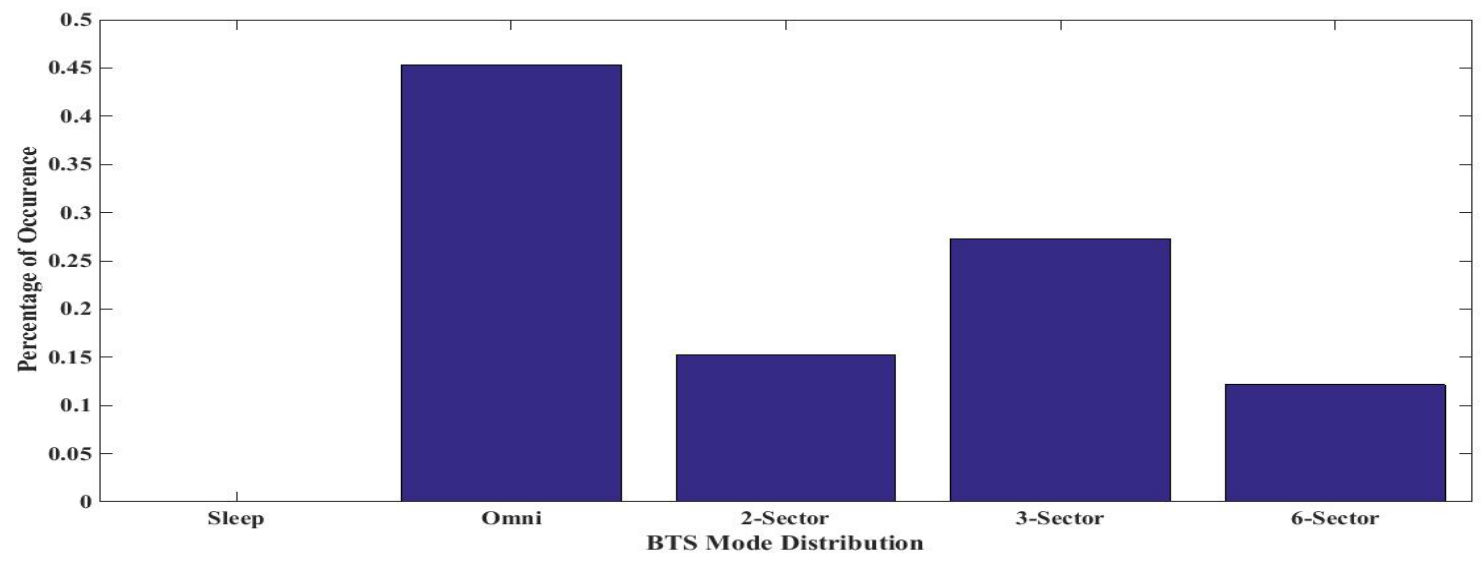

Figure 5.10: BTS Mode Distribution. Max $\lambda=1000$, Transition Cost $=0$, Delay Cost $=$ 100, Epoch $=1 \mathrm{~s}$

\subsection{Action Distribution Analysis}

\subsubsection{Action Distribution Analysis Without Delay and Transition Cost}

Remaining in the same mode is the most prominent action during the operation of a BTS with MDP under normal condition, i.e., without delay and transition cost and time epoch of one second, as shown in Fig. 5.11. Actions that switch BTS to another mode, either upper mode or lower mode, are more or less equal but significantly less in comparison to the action that keeps the BTS mode unchanged. 


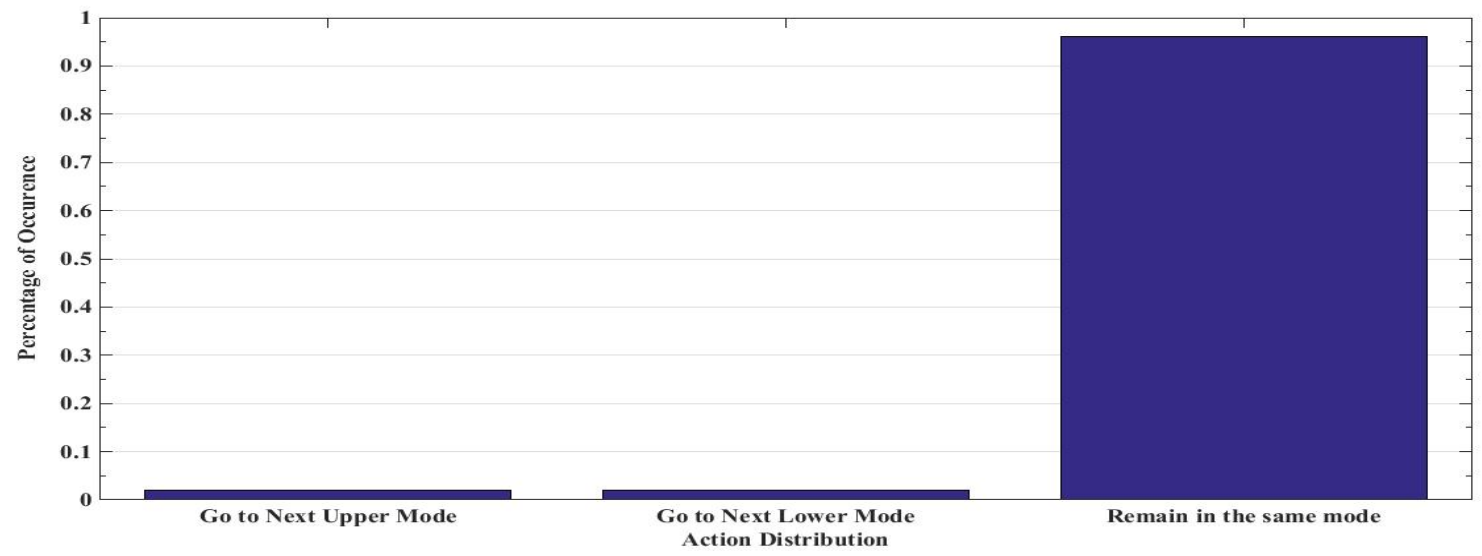

Figure 5.11: Action Distribution. Max $\lambda=1000$, Transition Cost $=0$, Delay Cost $=0$, Epoch $=1 \mathrm{~s}$

\subsubsection{Action Distribution Analysis With Transition Cost}

As expected, action distribution is fully inclined towards the action that refuses to change the operating mode of BTS in line with variable traffic load as shown in Fig. 5.12.

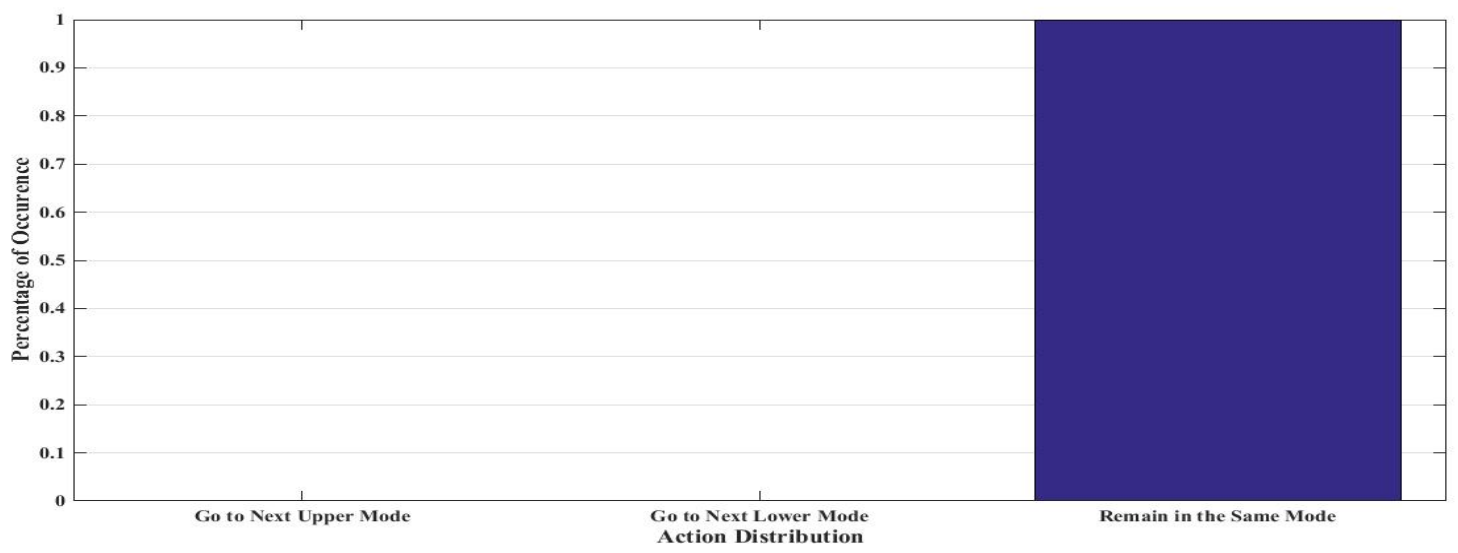

Figure 5.12: Action Distribution Analysis. Max $\lambda=1000$, Transition Cost $=1000$, Delay Cost $=0$, Epoch $=1 \mathrm{~s}$

This is because the system opts to stay in its current mode rather than incurring the cost to change mode, either towards upper mode or lower mode. 


\subsubsection{Action Distribution Analysis With Delay Cost}

Action distribution is more distributed towards actions that switch operating modes when delay cost is introduced as can be seen from Fig. 5.13. In contrast to normal operating condition, the share of action that keeps the current mode unchanged is decreased a bit while actions that switch the BTS mode to higher mode or lower mode are increased.

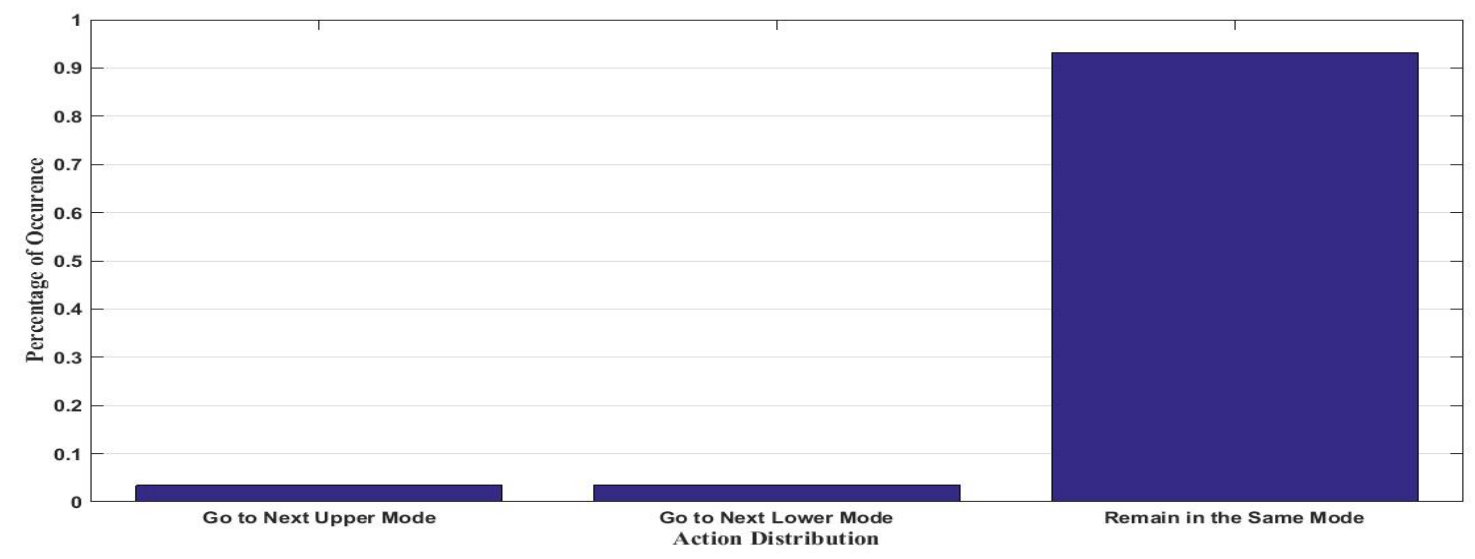

Figure 5.13: Action Distribution. Max $\lambda=1000$, Transition Cost $=0$, Delay Cost $=100$, Epoch $=1 \mathrm{~s}$

\subsection{Integrated Analysis}

BTS operation management in basic arrangement, i.e., without delay and transition cost and time epoch of one second, can be taken as the benchmark arrangement for BTS operation. In this setup, we can see significant difference between the BTS operation in terms of power consumption with and without MDP as shown in Fig. 5.2. As such, significant power saving between $41 \%$ and $83 \%$ has been observed as shown in Fig. 5.3. This proves the efficacy of our proposed model for BTS operation to save energy consumption. Mode distribution shows that the BTS operates in sleep mode or 6-Sector mode rarely in this benchmark arrangement as shown in Fig. 5.8. Likewise, in terms of action distribution, action that avoids mode change comprises approximately $95 \%$ of action whereas actions that switches 
modes to upper mode or lower mode both comprise merely $5 \%$.

When transition cost is introduced into BTS operation, the power consumption and power saving graph show the similar pattern. 3-sector and 2-sector modes are the most prominent modes whereas BTS switching actions are negligible in comparison to action that keeps BTS in its current mode of operation, as system responds as expected to transition cost, i.e., mode switching cost. However, most significant is the reduction in the power saving, i.e., almost $80 \%$ reduction is observed in this combination during low traffic times in comparison to the benchmark arrangement. However, the system can take advantage by avoiding losses it has to incur in terms of handover and other switching related costs. Further, careful consideration must be given while choosing the transition cost as close to actual cost, so that the system does not choose to stay at it's current mode thereby degrading the quality of system operation.

In comparison to the previous two arrangements of BTS operation, the arrangement in which delay cost is introduced, shows some interesting observations. Most importantly, the power saving is in between the two arrangements discussed above with and without transition cost for most of the day. The power saving is reduced during high traffic arrival time as the system moves to 6-sector mode of operation quite often. When the BTS switches to 6-sector mode often, energy consumption is the same irrespective of the system is using MDP or not. Likewise, this arrangement also shows frequent switching between modes in comparison to other arrangement as indicated by high share of actions that change the modes of operation of a BTS. This is also supported by the operation of BTS modes in all modes, except in sleep mode. The loss of power saving in comparison to benchmark arrangement is the improvement in quality of service of the BTS operation by supporting higher number of channel availability. It is supported by the fact that in this set-up, BTS frequently moves to 6-Sector mode, hence no more waiting for the incoming call request, which can be served immediately. 


\subsection{Analysis on Effect of Epoch Time}

Fig. 5.14 shows the variation in total power saving when epoch time is varied. The small epoch time saves higher amount of power in comparison to the larger epoch time. This is clearly seen for higher arrival rate than low arrival rate. The power saving is high at 1 second epoch time and low at epoch time of 10 seconds. As expected, small epoch time increases the system's ability to respond to traffic variation much faster than that of larger epoch time. It shows that when the network can respond faster to time varying traffic, higher power savings can be achieved. However, this comes at the cost of switching cost. Network cannot instantly switch between modes due to the time spent in making physical adjustments to the network. Likewise, the epoch time cannot be shorter than the time required by the system to switch between modes.

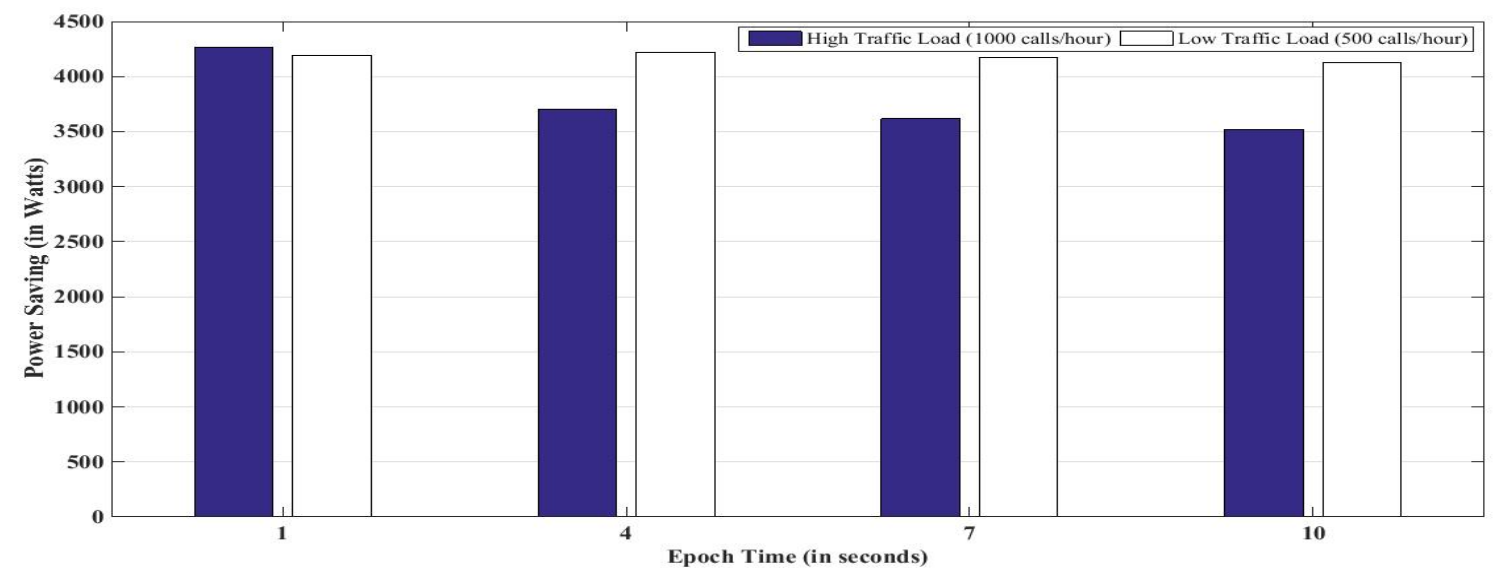

Figure 5.14: Total Power Saving Variation against Time Epochs 


\section{Chapter 6}

\section{Conclusions and Future Works}

\subsection{Summary}

ICT sector consumes electricity as its main supply of energy and this electricity comes from different sources, especially through burning of fossil fuel. This burning of fossil fuel is contributing to the greenhouse gas (GHG) emission. Hence, it is today's research trend to find ways to minimize energy consumption in ICT sector not only to reduce OpEx of the operators and users alike but also to reduce the GHG emission by this sector.

Talking about ICT sector, the focus of this study is on the energy consumption minimization of the BTS, which is one of the most energy hungry but critical component of the access network of a cellular telecommunication system. Several methods have been proposed through research to achieve this, but at this point in time, this is still in progress to achieve best solutions as majority of the research keep on complimenting each other to find an optimal solution. Following that, an MDP optimisation technique has been proposed to achieve energy optimized operation of a BTS.

As required by the MDP optimization tool, the basic tuples of a MDP process viz. states, actions, transition probability matrix, rewards, time epoch, and discount factor have been defined to correctly capture the dynamics of the birth-death process inside the BTS in a cellular communication network. The optimal policy thus identified by the MDP has been 
used to operate the BTS dynamically, i.e., turning ON/OFF the appropriate sectors of the BTS site, against the variable traffic load so that the BTS will operate in an energy efficient manner in a long term. The effect of BTS mode switching has also been taken into account in the form of transition cost, which is the lump sum cost that represents the cost of user handover, necessary arrangement of sector configuration, processing time to switch between modes and other factors. Likewise, delay cost has also been dealt with that reduces the overall reward obtained through MDP process in order to make the system look into QoS issue as well when looking to minimize energy consumption. This cost forces the network controller to open new sector at the cost of high energy consumption. Finally, the effect of epoch time on the overall power saving has been studied and presented.

Detailed analysis has proven that MDP as an optimization tool is useful in minimizing the overall energy consumption of a BTS network in spite of the transition and delay cost associated with the system operations. Further, it is vital that the efficiency of the proposed model depends largely on capturing the real environment as accurately as possible.

The main contributions and future work in addition to this work has been detailed in the next sections.

\subsection{Main Contributions}

The main contributions of our work are illustrated as below:

- Optimization of BTS operation management in an energy efficient way is formulated as a MDP problem. The solution to this problem is the MDP policy that guides the network controller to take appropriate action specific to the network state so that long term average energy consumption is minimized.

- MDP policy guides the energy efficient operation of a BTS. In this study, such operation is carried out by altering the sectorization of the BTS site. While doing so, user handover between turning ON sector and turning OFF sector has to be carried out, necessary antenna configuration has to be done, extra processing time other than the normal 
operation of the BTS operation is required, and other factors that impact the smooth operation of the BTS. This cost is significant and cannot be neglected. The impact of this effect is taken into account as a lump sum cost which reduces the actual reward when state transition takes place. The impact of such cost on actual energy saving has been studied and presented.

- Furthermore, even though the system is equipped with enough resource to address the peak time traffic, due to the inherent nature of the MDP policy to operate on maximizing the long term reward of the system, the system might sometime not react to the immediate traffic demand, thus undermining the quality of the system. Hence, delay cost has been introduced to address this concern where the actual reward will be decreased when the call request is delayed. Effect of delay cost in the system has been analysed and presented.

- Epoch time, the time that the network controller chooses to inspect the state of the system and take appropriate decision, is vital to the operation of the BTS in an energy efficient manner. Too short and too long epoch time impact the system operation in their own way, which has been analysed in the study.

\subsection{Future Works}

Probable future research works which have not been considered in this study that might be supplement to this study have been detailed as below:

- This study has considered only a single BTS site. It would be a good idea to consider the multiple tier of cells so that the overall picture of energy consumption can be obtained in a certain geographical area considering the traffic movement within such area.

- Single and homogeneous type of traffic distribution has been considered in this study. It would be interesting to consider multiple traffic classes as well as spatially varying traffic so that more insight into real energy saving can be realized. 
- Distributed network controllers residing in BTS site without coordination with other BTS site has been considered in this study. Centralized controller coordinating between eNBs, i.e., BTSs, can be an interesting research topic.

- Grouped state space has been considered in this study so as to reduce computational complexity. However, defining single channel occupancy as single state space in MDP formulation can be another topic of research interest.

- Maximum of six-sector sectorization has been considered in this study which can be increased to 12 sectors. Such higher order sectorisation might be helpful in identifying if that is really energy efficient or not even though it is expected to increase data rate per unit area.

- Fixed switching cost is assumed in this study. It would be interesting to analyse the system operation with variable switching cost that depends on spatial and temporal statistics. 


\section{Appendix A}

\section{Abbreviation List}

$\begin{array}{ll}\text { 3GPP } & 3^{\text {rd }} \text { Generation Partnership Project } \\ \text { APC } & \text { Area Power Consumption } \\ \text { BTS } & \text { Base Transceiver Station } \\ \text { CapEx } & \text { Capital Expenditure } \\ \text { DTMDP } & \text { Discrete Time Markov Decision Process } \\ \text { ECR } & \text { Energy Consumption Ratio } \\ \text { eNB } & \text { Evolved Node B } \\ \text { EARTH } & \text { Energy Aware Radio and neTwork tecHnologies } \\ \text { FMDP } & \text { Finite Markov Decision Process } \\ \text { GHG } & \text { Greenhouse Gas } \\ \text { GoS } & \text { Grade of Service } \\ \text { GSM } & \text { Global Systems of Mobile Communication } \\ \text { ICT } & \text { Information and Communication Technology } \\ \text { ITU } & \text { International Telecommunication Union } \\ \text { LTE-A } & \text { Long Term Evolution Advanced } \\ \text { MDP } & \text { Markov Decision Process } \\ \text { NSS } & \text { Network and Switching Subsystem } \\ \text { OpEx } & \text { Operational Expenditure } \\ \text { QoS } & \text { Quality of Service } \\ \text { RBs } & \text { Resource Blocks } \\ \text { RSS } & \text { Radio Subsystem } \\ \text { SON } & \text { Self-organizing Network } \\ \text { TPC } & \text { Throughput Power Consumption } \\ \text { TPM } & \text { Transition Probability Matrix } \\ & \end{array}$




\section{Bibliography}

[1] Y. S. Soh, T. Q. S. Quek, M. Kountouris, and H. Shin, "Energy Efficient Heterogeneous Cellular Networks", IEEE Journal on Selected Areas in Communications, vol. 31, no. 5, pp. 840-850, May. 2013.

[2] D. Lister, Vodafone Group Research \& Development, "An operator's view on green radio", presented at the Proc. IEEE International Workshop on Green Communications, Jun. 2009.

[3] M. Gupta, and S. Singh, "Greening of the Internet", ACM SIGCOMM 2003, Karlsruhe, Germany, Aug. 2003.

[4] W. Mohr, "The WINNER (wireless world initiative new radio) project-development of a radio interface for systems beyond 3G", presented at the IEEE 16th International Symposium on Personal, Indoor and Mobile Radio Communications (PIMRC 2005), vol. 2, pp. 857-862, Jun. 2005.

[5] EARTH, "Energy aware radio and network technologies project", https://www.ictearth.eu/

[6] C. Han, T. Harrold, S. Armour, I. Krikidis, S. Videv, P. M. Grant, H. Haas, J. S. Thompson, I. Ku, C. Wang, T. A. Le, M. R. Nakhai, J. Zhang, and L. Hanzo, "Green radio: radio techniques to enable energy-efficient wireless networks", IEEE Communications Magazine, vol. 49, no. 6, pp. 46-54, Jun. 2011. 
[7] OPERA-Net, "Optimising power efficiency in mobile radio networks project", https://www.celticplus.eu/project-operanet/, accessed on November 03, 2016.

[8] eWIN, "Energy-efficient WIreless Networking", https://www.kth.se, accessed on November 03, 2016.

[9] 3GPP, "3rd Generation Partnership Project", www.3gpp.org, accessed on November 03, 2016.

[10] M. A. Marsan, C. Guerrero, S. Buzzi, F. Idzikowski, L. Chiaraviglio, M. Meo, Y. Ye, and J. L. Vizcaino, "TREND: Toward real energy-efficient network design", Sustainable Internet and ICT for Sustainability (SustainIT), pp. 1-6, Oct. 2012.

[11] D. Liu, and C. Yang, "Energy Efficiency of Downlink Networks With Caching at Base Stations" IEEE Journal on Selected Areas in Communications, vol. 34, no. 4, pp. 907922, Apr. 2016.

[12] J. Wu, Y. Zhang, M. Zukerman, and E. K.Yung, "Energy-Efficient Base-Stations SleepMode Techniques in Green Cellular Network: A Survey" IEEE Communication Surveys and Tutorials, vol. 17, no. 2, pp. 803-826, Second Quarter 2015.

[13] Z. Niu, Y. Wu, J. Gong, and Z. Yang, "Cell Zooming for cost-efficient green cellular networks", IEEE Comunications Magazine, vol. 48, no. 11, pp. 74-79, Nov. 2010.

[14] R. Balasubramaniam, S. Nagaraj, M. Sarkar, C. Paolini, and P. Khaitan, "Cell zooming for power efficient base station operation" 9th International Wireless Communications and Mobile Computing Conference (IWCMC), pp. 556-560, Jul. 2013.

[15] E. Oh, K. Son, and B. Krishnamachari, 'Dynamic Base Station Switching-On/Off Strategies for Green Cellular Networks" IEEE Transactions on Wireless Communications, vol. 12, no. 5, pp. 2126-2136, May 2013. 
[16] N. Yu, Y. Miao, L. Mu, H. Du, H. Huang, and X. Jia, 'Minimizing Energy Cost by Dynamic Switching ON/OFF Base Stations in Cellular Networks" IEEE Transactions on Wireless Communications, vol. 15, no. 11, pp. 7457-7469, Nov. 2016.

[17] K. Son, R. Guruprasad, S. Nagaraj, M. Sarkar, and S. Dey, 'Dynamic cell reconfiguration framework for energy conservation in cellular wireless networks" Journal of Communications and Networks, vol. 18, no. 4, pp. 567 - 579, Aug. 2016.

[18] C. Liu, B. Natarajan, and H. Xia, 'Small Cell Base Station Sleep Strategies for Energy Efficiency" IEEE Transactions on Vehicular Technology, vol. 65, no. 3, pp. 1652 - 1661, Mar. 2016.

[19] L. Li, M. Peng, C. Yang and Y. Wu, 'Optimization of Base-Station Density for High Energy-Efficient Cellular Networks With Sleeping Strategies" IEEE Transactions on Vehicular Technology, vol. 65, no. 9, pp. 7501 - 7514, Sep. 2016.

[20] J. Kim, W. S. Jeon, and D. G. Jeong, 'Base-Station Sleep Management in Open-Access Femtocell Networks" IEEE Transactions on Vehicular Technology, vol. 65, no. 5, pp. 3786 - 3791, May. 2016.

[21] R. Li, Z. Zhao, X. Chen, J.Palicot, and H. Zhang, "TACT: A Transfer Actor-Critic Learning Framework for Energy Saving in Cellular Radio Access Networks" IEEE Transactions on Wireless Communications, vol. 13, no. 4, pp. 2000-2011, Apr. 2014.

[22] H. Holma, A. Toskala, "LTE for UMTS: Evolution to LTE-Advanced", Second Edition, John Wiley \& Sons, West Sussex, 2011.

[23] L. G. Hvizi, and I. Gdor, "Power Savings in Mobile Networks by Dynamic Base Station Sectorization" 2011 IEEE 22nd International Symposium on Personal, Indoor and Mobile Radio Communications, pp. 2415-2417, Sep. 2011. 
[24] M. F. Hossain, K. S. Munasinghe, and A. Jamalipour, "Energy-Aware Dynamic Sectorization of Base Stations in Multi-Cell OFDMA Networks" IEEE Wireless Communications Letters, vol. 2, no. 6, pp. 587-590, Dec. 2013.

[25] EARTH, "INFSO-ICT-247733 EARTH Deliverable D6.4, Final Integrated Concept", Jul. 2012.

[26] Y. Qi, M. A. Imran, and R. Tafazolli, "Energy-Aware Adaptive Sectorisation in LTE Systems" IEEE 22nd International Symposium on Personal, Indoor and Mobile Radio Communications, pp. 2402-2406, 2011.

[27] D. Quintas, and V. Friderikos, "On Dynamic Policies to Switch Off Relay Nodes" IEEE International Conference on Communications (ICC), pp. 2945-2950, Jun. 2012.

[28] Z. H. Abbas, and F. Y. Li, "Energy Optimization in Cellular Networks with Micro/Pico cells using Markov Decision Process" 18th European Wireless Conference, Apr. 2012.

[29] J. Kim, P. Kong, N. Song, J. K. Rhee, and S. Al-Araji, "MDP Based Dynamic Base Station Management for Power Conservation in Self-Organizing Networks" Wireless Communications and Networking Conference (WCNC), Apr. 2014.

[30] W. Guo, J. M. Rigelsford, K. L. Ford, and T. O'Farrell "Dynamic Basestation Antenna Design for Low Energy Networks" Progress In Electromagnetics Research C, vol. 31, pp. 153-168, 2014.

[31] R. E. Bellman, "A Markovian Decision Process" Indiana University Mathematics Journal, vol. 6, no. 4, pp. 679-684, 1957.

[32] M. L. Puterman, "Markov decision processes: Discrete stochastic dynamic programming", John Wiley \& Sons, Hoboken NJ, 2005.

[33] Normalized cellular traffic trace during one week, this data was obtained from experiments conducted by the University of Southern Californias Autonomous Networks Research Group, http://anrg.usc.edu. 
[34] J. D. Gadze, S. B. Aboagye, and K. A. P. Agyekum, "Real Time Traffic Base Station Power Consumption Model for Telcos in Ghana", International Journal of Computer Science and Telecommunications, vol. 7, no. 5, pp. 6-13, Jul. 2016.

[35] R. G. Gallager, "Stochastic Processes: Theory for Applications", Cambridge University Press, Cambridge, 2013.

[36] H. Holtkamp, G. Auer, V. Giannini, and H. Haas, "A Parameterized Base Station Power Model", IEEE Communications Letters, vol. 17, no. 11, pp. 2033-2035, Nov. 2013.

[37] H. Holtkamp, G. Auer, S. Bazzi, and H. Haas, "Minimizing base station power consumption", IEEE Journal on Selected Areas in Communications, vol. 32, no. 2, pp. 297-306, Feb. 2014.

[38] G. Auer, V. Giannini, C. Desset, I. Godor, P. Skillermark, M. Olsson, M. A. Imran, D. Sabella, M. J. Gonzalez, O. Blume, and A. Fehske, "How much energy is needed to run a wireless network?", IEEE Wireless Communications,, vol. 18, no. 5, pp. 40-49, Oct. 2011.

[39] O. Arnold, F. Richter, G. Fettweis, and O. Blume, "Power Consumption Modeling of Different Base Station Types in Heterogeneous Cellular Networks", Future Network and Mobile Summit, pp. 1-8, 2010.

[40] K. Son, H. Kim, Y. Yi, and B. Krishnamachari "Base Station Operation and User Association Mechanisms for Energy-Delay Tradeoffs in Green Cellular Networks", IEEE Journal on Selected Areas in Communications, vol. 29, no. 8, pp. 1525-1536, Sep. 2011.

[41] I. Chades, G. Chapron, M.J. Cros, F. Garcia, and R. Sabbadin, MDPtoolbox: a multiplatform toolbox to solve stochastic dynamic programming problems. Ecography 37:916920., 2014. 